\title{
THE VARIETIES OF SEMI-CONFORMAL VECTORS OF AFFINE VERTEX OPERATOR ALGEBRAS
}

\author{
YANJUN CHU AND ZONGZHU LIN
}

\begin{abstract}
This is a continuation of our work to understand vertex operator algebras using the geometric properties of varieties attached to vertex operator algebras. For a class of vertex operator algebras including affine vertex operator algebras associated to a finite dimensional simple Lie algebra $\mathfrak{g}$, we describe their varieties of semi-conformal vectors by some matrix equations. These matrix equations are too complicated to be solved for us. However, for affine vertex operator algebras associated to the simple Lie algebra $\mathfrak{g}$, we find the adjoint group $G$ of $\mathfrak{g}$ acts on the corresponding varieties by a natural way, which implies that such varieties should be described more clearly by studying the corresponding $G$-orbit structures. Based on above methods for general cases, as an example, considering affine vertex operator algebras associated to the Lie algebra $\mathfrak{s l}_{2}(\mathbb{C})$, we shall give the decompositions of $G$-orbits of varieties of their semi-conformal vectors according to different levels. Our results imply that such orbit structures depends on the levels of affine vertex operator algebras associated to a finite dimensional simple Lie algebra $\mathfrak{g}$.
\end{abstract}

\section{INTRODUCTION}

1.1. This is a continuation of study of vertex operator algebras using the geometry of the variety of semi-conformal vectors, started in [JL2] and [CL].

Given a verex operator algebra $(V, Y, \mathbf{1}, \omega)$, a vertex operator subalgebra $\left(U, Y, \mathbf{1}, \omega^{\prime}\right)$ in general has a different conformal vector $\omega^{\prime} \neq \omega$. The subalgebra $U$ (respectively, $\omega^{\prime}$ ) is called semi-conformal if $\left.\omega_{n}^{\prime}\right|_{U}=\left.\omega_{n}\right|_{U}$ for $n \geq 0$ using the notation $Y(v, z)=\sum_{n} v_{n} z^{-n-1}$ for all $v \in V$. It follows from the definition $\omega^{\prime} \in V_{2}$. The $\operatorname{set} \operatorname{Sc}(V, \omega)$ of all semi-conformal vectors is a Zariski closed subset of $V_{2}([\mathrm{CL}$, Theorem 1.1]).

In [CL], we use the geometry of the set of semi-conformal vectors together with the relations of the corresponding subalgebras to give two characterizations of Heisenberg vertex operator algebras. In the Heisenberg algebra case, the finite dimensional abelian Lie algebra $\mathfrak{h}$ is equipped with a non-degenerated symmetric bilinear form. The automorphism group of $\mathfrak{h}$ (preserving the form) is the complex orthogonal group which acts on the set

2010 Mathematics Subject Classification. 17B69. 
$\operatorname{Sc}(V, \omega)$. In this paper, we study the geometric structures of $\operatorname{Sc}(V, \omega)$ for affine vertex operator algebras $V=V_{\widehat{\mathfrak{g}}}(\ell, 0)$ and $L_{\widehat{\mathfrak{g}}}(\ell, 0)$, where $L_{\widehat{\mathfrak{g}}}(\ell, 0)$ is the simple quotient of $V_{\widehat{\mathfrak{g}}}(\ell, 0)$, associated to a finite dimensional simple Lie algebra $\mathfrak{g}$. In this case, the adjoint group $G$ of $\mathfrak{g}$ also acts on the variety $\operatorname{Sc}(V, \omega)$. We shall describe $G$-orbit structures.

1.2. Denoted by $\operatorname{ScAlg}(V, \omega)$ the set of semi-conformal subalgebras of a vertex operator algebra $V$ with conformal vector $\omega$. Obviously, $\left(U, \omega^{\prime}\right) \longmapsto \omega^{\prime}$ defines a surjection from $\operatorname{ScAlg}(V, \omega)$ to $\operatorname{Sc}(V, \omega)$, denoted it by $\pi$. The commutant of a subalgebra in $V$ gives an operator $C_{V}$ on $\operatorname{ScAlg}(V, \omega)$ as follows

$$
\begin{aligned}
C_{V}: \operatorname{ScAlg}(V, \omega) & \longrightarrow \operatorname{ScAlg}(V, \omega) \\
\left(U, \omega^{\prime}\right) & \longmapsto\left(C_{V}(U), \omega-\omega^{\prime}\right) .
\end{aligned}
$$

And $C_{V}$ induces an involution operator $\omega-$ on $\operatorname{Sc}(V, \omega)$ defined by $\omega^{\prime} \longmapsto \omega-\omega^{\prime}$. In fact, these maps form a following commutative diagram

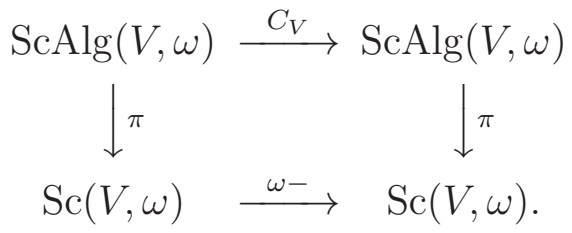

Semi-conformal subalgebras and semi-conformal vectors are used to study level-rank duality in vertex operator algebras ([JL2]). Such subalgebras are motivated by [LL, Theorem 3.11.12] and [FZ, Theorem 5.1] to determine duality pairs of vertex operator subalgebras in Howe duality [LL1, CHZ], and depend on coset constructions of vertex operator algebras.

1.3. For the surjection $\pi$, the fiber $\pi^{-1}\left(\omega^{\prime}\right)$ for each $\omega^{\prime} \in \operatorname{Sc}(V, \omega)$ is the set of semiconformal subalgebras with conformal vector $\omega^{\prime}$ of $V$. In $\pi^{-1}\left(\omega^{\prime}\right)$, there exists a unique maximal one $U\left(\omega^{\prime}\right)$, which is the maximal conformal extension (in $V$ ) of semi-conformal subalgebras with conformal vector $\omega^{\prime}$ and can be realized as the double commutant $C_{V}\left(C_{V}\left(\left\langle\omega^{\prime}\right\rangle\right)\right)$ of $\left\langle\omega^{\prime}\right\rangle$ in $V$, where $\left\langle\omega^{\prime}\right\rangle$ is the Virasoro vertex operator algebra generated by $\omega^{\prime}$. In fact, semi-conformal vectors classify semi-conformal subalgebras of $V$ up to conformal extension. Thus, for each $\omega^{\prime} \in \operatorname{Sc}(V, \omega)$, by studying the fiber $\pi^{-1}\left(\omega^{\prime}\right)$, we can classify the set $\operatorname{ScAlg}(V, \omega)$. For $\omega^{\prime}, \omega^{\prime \prime} \in \operatorname{Sc}(V, \omega)$, we define a partial order $\omega^{\prime} \preceq \omega^{\prime \prime}$ if $U\left(\omega^{\prime}\right) \subseteq U\left(\omega^{\prime \prime}\right)([\mathrm{CL}$, Definition 2.7]). In Heisenberg algebra case, we described the structure of $\operatorname{Sc}(V, \omega)$ as $G=\operatorname{Aut}(V, \omega)$-orbits. Moreover, for a vertex operator algebra $(V, \omega)$, when $\operatorname{Sc}(V, \omega)$ satisfies some properties, we characterized $(V, \omega)$ as a Heisenberg vertex operator algebra with conformal vector $\omega_{0}$ (cf. [CL, Theorem 1.4 and Theorem 1.5]). We also note that the group $G=\operatorname{Aut}(V, \omega)$ acts on both $\operatorname{ScAlg}(V, \omega)$ and $\operatorname{Sc}(V, \omega)$ and the $\operatorname{map} \pi$ is $G$-equivariant. 
1.4. For affine vertex operator algebras $V=V_{\widehat{\mathfrak{g}}}(\ell, 0)$ and $L_{\widehat{\mathfrak{g}}}(\ell, 0)$ associated to a finite dimensional simple Lie algebra $\mathfrak{g}$, we choose an orthonormal basis of the Lie algebra $\mathfrak{g}=$ $V_{1}$. Then a vector belongs to $\operatorname{Ker} L(1) \cap V_{2}$ if and only if there exists a symmetric matrix such that it can be expressed as a quadratic form with respect to the given orthonormal basis of $\mathfrak{g}$ (See Proposition 3.1 in Section 3). This proposition is critical for us to find semi-conformal vectors of $(V, \omega)$. With the help of this proposition, we characterize semiconformal vectors of $(V, \omega)$ by some matrix equations (See equations (3.4)-(3.8) in Section 3). But these matrix equations obtained are too complicate to be solved in general. As Heisenberg case indicated in $\mathrm{CL}$, the variety $\operatorname{Sc}(V, \omega)$ is closely related to the affine Lie algebra with a fixed level as well as the lattice structure. For affine vertex operator algebras $V=V_{\widehat{\mathfrak{g}}}(\ell, 0)$ and $L_{\widehat{\mathfrak{g}}}(\ell, 0)$, considering the action of the adjoint group $G$ of $\mathfrak{g}$ on the variety $\operatorname{Sc}(V, \omega)$, we expect to give decompositions of $G$-orbits of $\operatorname{Sc}(V, \omega)$.

To get $G$-orbits of $\operatorname{Sc}(V, \omega)$ for affine vertex operator algebras $V=V_{\widehat{\mathfrak{g}}}(\ell, 0)$ and $L_{\widehat{\mathfrak{g}}}(\ell, 0)$ associated to a finite dimensional simple Lie algebra $\mathfrak{g}$, in this paper, we shall consider affine vertex operator algebras $V_{\widehat{\mathfrak{s l}}_{2}}(\ell, 0)$ and $L_{\widehat{\mathfrak{s l}}_{2}}(\ell, 0)$ as examples. At first, we study the variety $\operatorname{Sc}(V, \omega)$ for $V=V_{\widehat{\mathfrak{s}}_{2}}(\ell, 0)$. When the matrix equations (3.4)-(3.8) in Section 3 are applied to $V_{\widehat{\mathfrak{s}}_{2}}(\ell, 0)$, we can obtain matrix equations describing $\operatorname{Sc}\left(V_{\widehat{\mathfrak{s l}}_{2}}(\ell, 0), \omega\right)$. On the other hand, since the adjoint group $\mathrm{PSL}_{2}(\mathbb{C})$ of $\mathfrak{s l}_{2}(\mathbb{C})$ is isomorphic to the special orthogonal group $\mathrm{SO}_{3}(\mathbb{C})$, then the $\mathrm{PSL}_{2}(\mathbb{C})$-action on $\operatorname{Sc}\left(V_{\widehat{\mathfrak{s}}_{2}}(\ell, 0), \omega\right)$ can be realized by the group $\mathrm{SO}_{3}(\mathbb{C})$ in a natural way. Moreover, we find that the orbits of $\operatorname{Sc}\left(V_{\mathfrak{s}_{2}}(\ell, 0), \omega\right)$ are determined completely by solutions with diagonal matrices in above mentioned matrix equations. Thus, the problems are reduced to find all solutions with the form of diagonal matrices from obtained matrix equations. Finally, we obtain the decomposition of $\mathrm{PSL}_{2}(\mathbb{C})$-orbits of the varieties $\operatorname{Sc}\left(V_{\widehat{\mathfrak{s l}}_{2}}(\ell, 0), \omega\right)$ for the level $\ell \neq-2$. Such decompositions can be summarized as follows

1) If $\ell=0,1$, then $\operatorname{Sc}\left(V_{\widehat{\mathfrak{s l}}_{2}}(\ell, 0), \omega\right)=\{0\} \cup\{\omega\}$;

2) If $\ell \neq-2,0,1,4$, then $\operatorname{Sc}\left(V_{\widehat{\mathfrak{s l}}_{2}}(\ell, 0), \omega\right)=\operatorname{Orb}_{\omega_{A_{1}}} \cup \operatorname{Orb}_{\omega_{A_{4}}} \cup\{0\} \cup\{\omega\}$, where $\operatorname{Orb}_{\omega_{A}}=$ $\left\{\omega_{o A o^{t r}} \mid o \in \mathrm{SO}_{3}(\mathbb{C})\right\}$ and

$$
A_{1}=\left(\begin{array}{ccc}
\frac{1}{2 \ell} & 0 & 0 \\
0 & 0 & 0 \\
0 & 0 & 0
\end{array}\right), A_{4}=\left(\begin{array}{lll}
-\frac{1}{\ell(\ell+2)} & 0 & 0 \\
0 & \frac{1}{2(\ell+2)} & 0 \\
0 & 0 & \frac{1}{2(\ell+2)}
\end{array}\right)
$$

3) If $\ell=4$, then $\operatorname{Sc}\left(V_{\widehat{\mathfrak{s l}}_{2}}(\ell, 0), \omega\right)=\{0\} \cup\{\omega\} \cup \bigcup_{\omega_{A} \in T} \operatorname{Orb}_{\omega_{A}}$, where

$$
T=\left\{A=\left(\begin{array}{lll}
a_{1} & 0 & 0 \\
0 & a_{2} & 0 \\
0 & 0 & a_{3}
\end{array}\right) \mid \operatorname{tr}(A)=\frac{1}{8} \text { and } a_{1} a_{2}+a_{1} a_{3}+a_{2} a_{3}=0\right\} / S_{3} .
$$


These results shows that for $\ell \neq-2,0,1,4, \operatorname{Sc}\left(V_{\widehat{\mathfrak{s l}_{2}}}(\ell, 0), \omega\right)$ is the union of finitely many $\mathrm{PSL}_{2}(\mathbb{C})$-orbits and has two nontrivial $\mathrm{PSL}_{2}(\mathbb{C})$-orbits. However, $\operatorname{Sc}\left(V_{\widehat{\mathfrak{s l}}_{2}}(4,0), \omega\right)$ consists of infinitely many $\mathrm{PSL}_{2}(\mathbb{C})$-orbits. Moreover, for the simple quotients $\left(L_{\widehat{\mathfrak{s l}}_{2}}(\ell, 0), \omega\right)$ of $V_{\widehat{\mathfrak{s l}}_{2}}(\ell, 0)$ for the level $\ell \in \mathbb{Z}_{+}$, we also prove that they have the same decompositions as $\operatorname{Sc}\left(V_{\widehat{\mathfrak{s l}}_{2}}(\ell, 0), \omega\right)$.

1.5. Our results imply also some new problems to understand general affine vertex operator algebras and lattice vertex operator algebras. On one hand, since the vertex operator algebra $L_{\widehat{\mathfrak{s}}_{2}}(1,0)$ is isomorphic to the lattice vertex operator algebra $V_{A_{1}}$ associated to the root lattice of $A_{1}$ type. Then we know that $\operatorname{Sc}\left(V_{A_{1}}, \omega\right)$ consists of two trivial $\mathrm{PSL}_{2}(\mathbb{C})$ orbits $\{0\}$ and $\{\omega\}$. When we consider the orbit structures of $\operatorname{Sc}\left(L_{\widehat{\mathfrak{g}}}(1,0), \omega\right)$ for the cases of the simple affine vertex operator algebras $L_{\widehat{\mathfrak{g}}}(1,0)$ associated to the finite dimensional simple Lie algebras $\mathfrak{g}$ of $A, D, E$ types. We can study $G$-orbit informations of $\operatorname{Sc}(V, \omega)$ for the lattice vertex operator algebra $V_{Q_{\mathfrak{g}}}$ associated to the root lattice of $\mathfrak{g}$ because of $L_{\widehat{\mathfrak{g}}}(1,0) \cong V_{Q_{\mathfrak{g}}}$. On the other hand, it follows from above results that the $\mathrm{PSL}_{2}(\mathbb{C})$-orbit structure of $\operatorname{Sc}\left(V_{\widehat{\mathfrak{s l}}_{2}}(\ell, 0), \omega\right)$ depends on the level $\ell$, which leads us to think of some new problems such as, why has $\operatorname{Sc}\left(V_{\widehat{\mathfrak{s l}}_{2}}(\ell, 0), \omega\right)$ finitely or infinitely many $\mathrm{PSL}_{2}(\mathbb{C})$-orbits for different levels $\ell$ ? what is the level $\ell$ taken such that $\operatorname{Sc}\left(V_{\widehat{\mathfrak{g}}}(\ell, 0), \omega\right)$ has finitely or infinitely many $G$-orbits? Such problems can help us to understand deeply the affine vertex operator algebra $V$ by geometric properties of its varieties $\operatorname{Sc}(V, \omega)$.

1.6. By [JL2, Lemma. 5.1], a vertex subalgebra $U$ of $V$ cannot contain two different conformal vectors $\omega^{\prime}$ and $\omega^{\prime \prime}$ such that both $\left(U, \omega^{\prime}\right)$ and $\left(U, \omega^{\prime \prime}\right)$ are semi-conformal vertex operator subalgebras of $(V, \omega)$. We remark that on a vertex algebra, there can be many different conformal vectors to make it become non-isomorphic vertex operator algebras, even thought they have the same conformal gradations. The result of [JL2, Lem. 5.1] says that no one is a semi-conformal with respect to another. [BF, Example 2.5.9] provides a large number of such examples on the Heisenberg vertex operator algebras. Thus the map from $\operatorname{ScAlg}(V, \omega)$ to the set of all vertex subalgebras of $V$, forgetting the conformal structure, is injective. The conformal vector in $V$ uniquely determines the semi-conformal structure on a vertex subalgebra of $V$ if there is any.

One of the main motivations of this work is to investigate the conformal structure on a vertex subalgebra of a vertex operator algebra. In conformal field theory, the conformal vector completely determines the conformal structure (the module structure for the Virasoro Lie algebra). In mathematical physics, a vertex operator algebra has been investigated extensively as a Virasoro module (see [DMZ, D, KL, DLM, LY, M, LS, ,, L, Sh] by virtue of conformal vector. 
1.7. This paper is organized as follows: In Sect.2, we shall review the basic notions and results of semi-conformal subalgebras(vectors) for a vertex operator algebra according to [JL2, LL, CL; In Sect.3, we recall some related results of the affine vertex operator algebras associated to the finite dimensional simple Lie algebra $\mathfrak{g}$, and then give the description of the variety of semi-conformal vectors of $V_{\widehat{\mathfrak{g}}}(\ell, 0)$ by some matrix equations; Moreover, for affine vertex operator algebras $V_{\widehat{\mathfrak{g}}}(\ell, 0)$ and $L_{\widehat{\mathfrak{g}}}(\ell, 0)$, we shall study the action of the adjoint group $G$ of $\mathfrak{g}$ on varieties $\operatorname{Sc}\left(V_{\widehat{\mathfrak{g}}}(\ell, 0), \omega\right)$ and $\operatorname{Sc}\left(L_{\widehat{\mathfrak{g}}}(\ell, 0), \omega\right)$. In Sect.4, as examples, we shall study $\operatorname{PSL}_{2}(\mathbb{C})$-orbit structures of the varieties $\operatorname{Sc}\left(V_{\widehat{\mathfrak{s l}}_{2}}(\ell, 0), \omega\right)$ and $\operatorname{Sc}\left(L_{\widehat{\mathfrak{s}}_{2}}(\ell, 0), \omega\right)$ for $\ell \neq-2,4$; In Sect. 5, we shall describe $\mathrm{PSL}_{2}(\mathbb{C})$-orbits of the varieties $\operatorname{Sc}\left(L_{\widehat{\mathfrak{s l}}_{2}}(\ell, 0), \omega\right)$ and $\operatorname{Sc}\left(L_{\widehat{\mathfrak{s l}}_{2}}(\ell, 0), \omega\right)$ for $\ell=4$.

1.8. Notations: $\mathbb{C}$ is the field of complex number; $\mathbb{Z}$ is the set of integers; $\mathbb{Z}_{+}$(resp. $\mathbb{Z}_{-}$) is the set of positive (resp. negative) integers; $\mathbb{N}$ is the set of non-negative integers.

Acknowledgement: This work started when the first author was visiting Kansas State University from September 2013 to September 2014. He thanks the support by Kansas State University and its hospitality. The first author also thanks China Scholarship Council for their financial supports. The second author thanks C. Jiang for many insightful discussions. This work was motivated from the joint work with her. The second author also thanks Henan University for the hospitality during his visit in the summer of 2016, during which this work was carried out.

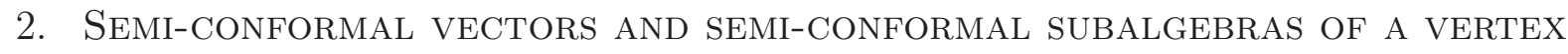 OPERATOR ALGEBRA}

2.1. For basic notions and results associated with vertex operator algebras, one is referred to the books [FLM, [LL, FHL, $\mathrm{BF}$. We will use $(V, Y, 1)$ to denote a vertex algebra and $(V, Y, 1, \omega)$ for a vertex operator algebra. When we deal with several different vertex algebras, we will use $Y^{V}, 1^{V}$, and $\omega^{V}$ to indicate the dependence of the vertex algebra or vertex operator algebra $V$. For example $Y^{V}\left(\omega^{V}, z\right)=\sum_{n \in \mathbb{Z}} L^{V}(n) z^{-n-2}$. To emphasize the presence of the conformal vector $\omega^{V}$, we will simply write $\left(V, \omega^{V}\right)$ for a vertex operator algebra and $V$ simply for a vertex algebra (with $Y^{V}$ and $1^{V}$ understood). We refer [B] for the concept of vertex algebras. Vertex algebras need not be graded, while a vertex operator algebra $\left(V, \omega^{V}\right)$ is always $\mathbb{Z}$-graded by the $L^{V}(0)$-eigenspaces $V_{n}$ with integer eigenvalues $n \in \mathbb{Z}$. We assume that each $V_{n}$ is finite dimensional over $\mathbb{C}$ and $V_{n}=0$ if $n<<0$.

2.2. In this section, we shall first review semi-conformal vectors (subalgebras) of a vertex operator algebra ([JL2, CL]). 
Let $V$ and $W$ be two vertex operator algebras with conformal vectors $\omega^{V}$ and $\omega^{W}$, respectively. Let $f: V \rightarrow W$ be a homomorphism of vertex algebras. Then $f$ is called conformal if $f \circ L^{V}(n)=L^{W}(n) \circ f$, for all $n \in \mathbb{Z}$, i.e., $f\left(\omega^{V}\right)=\omega^{W}$. We say $f$ is semiconformal if $f \circ L^{V}(n)=L^{W}(n) \circ f$, for all $n \geq 0$. Let $\left(V, \omega^{V}\right)$ be a vertex subalgebra of $\left(W, \omega^{W}\right)$ and the map $f: V \rightarrow W$ is the inclusion, we say $V$ is a conformal subalgebra of $W$ if $f$ is conformal ( $V$ has the same conformal vector with $W$ ). If $f$ is semi-conformal, then $\left(V, \omega^{V}\right)$ is called a semi-conformal subalgebra of $\left(W, \omega^{W}\right)$ and $\omega^{V}$ is called a semiconformal vector of $\left(W, \omega^{W}\right)$.

We remark that if $V$ is of CFT type, then $\omega^{\prime} \in V_{2}$ is semi-conformal if and only if $\omega^{\prime} \in \operatorname{Ker}\left(L^{V}(1)\right)$ and $L^{\prime}(0) \omega^{\prime}=2 \omega$.

2.3. For a vertex operator algebra $\left(W, \omega^{W}\right)$, we define

$\operatorname{ScAlg}\left(W, \omega^{W}\right)=\left\{\left(V, \omega^{V}\right) \mid\left(V, \omega^{V}\right)\right.$ is a semi-conformal subalgebra of $\left.\left(W, \omega^{W}\right)\right\}$;

$\operatorname{Sc}\left(W, \omega^{W}\right)=\left\{\omega^{\prime} \in W \mid \omega^{\prime}\right.$ is a semi-conformal vector of $\left.\left(W, \omega^{W}\right)\right\}$;

$\overline{\mathrm{S}\left(W, \omega^{W}\right)}=\left\{\left(V, \omega^{\prime}\right) \in \operatorname{ScAlg}\left(W, \omega^{W}\right) \mid C_{W}\left(C_{W}(V)\right)=V\right\}$,

where $C_{W}(V)$ is the commutant defined in [LL, 3.11]. A semi-conformal subalgebra $\left(U, \omega^{U}\right)$ of $W$ is called conformally closed if $C_{W}\left(C_{W}(U)\right)=U$ (see [JL2]). So the set $\overline{S\left(W, \omega^{W}\right)}$ consists of all conformally closed semi-conformal subalgebras of $\left(W, \omega^{W}\right)$.

It follows from the definition that there is a surjective map $\operatorname{ScAlg}\left(W, \omega^{W}\right) \rightarrow \operatorname{Sc}\left(W, \omega^{W}\right)$ by $\left(V, \omega^{V}\right) \mapsto \omega^{V}$. There is also a surjective map $\operatorname{ScAlg}\left(W, \omega^{W}\right) \rightarrow \overline{\mathrm{S}\left(W, \omega^{W}\right)}$ defined by $\left(V, \omega^{V}\right) \mapsto\left(C_{W}\left(C_{W}(V), \omega^{V}\right)\right.$. Thus, the restriction of the map $\operatorname{ScAlg}\left(W, \omega^{W}\right) \rightarrow$ $\operatorname{Sc}\left(W, \omega^{W}\right)$ to the set $\overline{\mathrm{S}\left(W, \omega^{W}\right)}$ is a bijection ([CL, Proposition 2.1]).

Let $\left(V, \omega^{V}\right)$ be a semi-conformal subalgebra of $\left(W, \omega^{W}\right)$. Then $\left(V, \omega^{V}\right)$ has a unique maximal conformal extension $\left(C_{W}\left(C_{W}(V)\right), \omega^{V}\right)$ in $\left(W, \omega^{W}\right)$ in the sense that if $\left(V, \omega^{V}\right) \subset$ $\left(U, \omega^{V}\right)$, then $\left(U, \omega^{V}\right) \subset\left(C_{W}\left(C_{W}(V)\right), \omega^{V}\right)$ ( see [LL, Corollary 3.11.14]).

2.4. Let $\left(W, \omega^{W}\right)$ be a general $\mathbb{Z}$-graded vertex operator algebra. The set $\operatorname{Sc}\left(W, \omega^{W}\right)$ forms an affine algebraic variety $([\mathrm{CL}$, Theorem 1.1]. In fact, a semi conformal vector $\omega^{\prime} \in W$ can be characterized by algebraic equations of degree at most 2 as described in [CL, Proposition 2.2]. The algebraic variety $\operatorname{Sc}\left(W, \omega^{W}\right)$ has also a partial order $\preceq$ (See [CL, Definition 2.7]), and this partial order can be characterized by algebraic equations in [CL, Proposition 2.8].

2.5. By [CL, Remark 2.10], to determine the space $\operatorname{ScAlg}(V, \omega)$, one needs to

(i) Determine $\operatorname{Sc}(V, \omega)$ and

(ii) For each $\omega^{\prime} \in \operatorname{Sc}(V, \omega)$, determine the set of conformal subalgebras of $C_{V}\left(C_{V}\left(\left\langle\omega^{\prime}\right\rangle\right)\right)$. 
The following lemma is straight forward to verify using the definition.

Lemma 2.1. Let $\phi:\left(V, Y^{V}, \mathbf{1}, \omega^{V}\right) \rightarrow\left(W, Y^{W}, \mathbf{1}, \omega^{W}\right)$ be a conformal vertex operator algebra homomorphism. For any vertex operator subalgebra $\left(U, Y, \mathbf{1}, \omega^{\prime}\right)$ of $V,\left(\phi(U), Y, \mathbf{1}, \phi\left(\omega^{\prime}\right)\right)$ is also a vertex operator subalgebra of $\left(W, Y^{W}, \mathbf{1}, \omega^{W}\right)$. Furthermore, $\left(\phi(U), Y, \mathbf{1}, \phi\left(\omega^{\prime}\right)\right)$ is a semi-conformal (resp.conformal) subalgebra of $\left(W, Y^{W}, \mathbf{1}, \omega^{W}\right)$ if $\left(U, Y, \mathbf{1}, \omega^{\prime}\right)$ is a semiconformal (resp.conformal) subalgebra of $\left(V, Y^{V}, \mathbf{1}, \omega^{V}\right)$.

Remark 2.2. The lemma has following two immediate consequences. The first consequence is that the conformal homomorphism $\phi$ restricts to a map $\operatorname{Sc}\left(V, \omega^{V}\right) \rightarrow \operatorname{Sc}\left(W, \omega^{W}\right)$. This means that the $\operatorname{Sc}\left(V, \omega^{V}\right)$ is functorial in the category of vertex operator algebra with conformal homomorphisms as morphisms. The second consequence is that $\operatorname{Sc}\left(V, \omega^{V}\right)$ and $\operatorname{Scalg}\left(V, \omega^{V}\right)$ are invariant under automorphism group. Let $G=\operatorname{Aut}\left(V, \omega^{V}\right)$ be the group of all conformal automorphisms of $\left(V, \omega^{V}\right)$. The lemma implies that $G$ acts on the above three sets and the projection map $\pi: \operatorname{ScAlg}\left(V, \omega^{V}\right) \rightarrow \operatorname{Sc}\left(V, \omega^{V}\right)$ is $G$-equivariant. Thus the question of determining $\operatorname{Sc}\left(V, \omega^{V}\right)$ can be reduced to determining the $G$-orbits in $\operatorname{Sc}\left(V, \omega^{V}\right)$. Also, the subgroup $G_{\omega^{\prime}}=\operatorname{Stab}_{G}\left(\omega^{\prime}\right)$ acts on the fiber $\pi^{-1}\left(\omega^{\prime}\right)$.

Note that for a vertex operator algebra $V=\oplus_{n \in \mathbb{Z}} V_{n}$ with $V_{n}=\left\{v \in V \mid L^{V}(0)(v)=\right.$ $n v\}$ being the eigenspace, the group $G$ also acts on each $V_{n}$ making $V_{n}$ into a finite dimensional $G$-module. If $\omega^{\prime} \in \operatorname{Sc}\left(V, \omega^{V}\right)$, then $L^{V}(0) \omega^{\prime}=L^{\prime}(0) \omega^{\prime}=2 \omega^{\prime}$. This implies that $\operatorname{Sc}\left(V, \omega^{V}\right) \subseteq V_{2}$. Furthermore, $L^{V}(1) \omega^{\prime}=L^{\prime}(1) \omega^{\prime}=0$ implies that $\operatorname{Sc}\left(V, \omega^{V}\right) \subseteq$ $\operatorname{ker}\left(L^{V}(1)\right) \cap V_{2}$. In particular, $\operatorname{ker}\left(L^{V}(1)\right) \cap V_{2}$ is a $G$-module. One would be interested in determining the $G$-module structure. Thus we have

Proposition 2.3. Let $\left(V, \omega^{V}\right)$ be a vertex operator algebra, then $\operatorname{Sc}\left(V, \omega^{V}\right)$ is a $G=$ Aut $(V)$-stable subset of $\operatorname{ker}\left(L^{V}(1)\right) \cap V_{2}$.

Lemma 2.4. Let $\left(V, \omega^{V}\right)$ and $\left(W, \omega^{W}\right)$ be two CFT-type vertex operator algebras, respectively. If $\rho$ is a conformal vertex operator algebra homomorphism from $V$ to $W$, then $\rho$ induces a map from $\operatorname{Sc}\left(V, \omega^{V}\right)$ to $\operatorname{Sc}\left(W, \omega^{W}\right)$, denoted by $\widehat{\rho}$. Thus, we have

1) If $\operatorname{Ker} \rho \cap \operatorname{Sc}\left(V, \omega^{V}\right)=\{0\}$, then $\widehat{\rho}$ is an injective map ;

2) If $\rho$ is surjective and $\operatorname{Ker} \rho \cap\left(V_{1} \oplus V_{2}\right)=\{0\}$, then $\widehat{\rho}$ is an isomorphism from $\operatorname{Sc}\left(V, \omega^{V}\right)$ to $\operatorname{Sc}\left(W, \omega^{W}\right)$.

Proof. The conformal vertex operator algebra homomorphism $\rho$ is a gradation-preserving map and it satisfies

$$
\begin{gathered}
\rho\left(1_{V}\right)=1_{W} ; \rho\left(\omega^{V}\right)=\omega^{W} \\
\rho\left(Y_{V}(v, z) u\right)=Y_{W}(\rho(v), z) \rho(u), \text { for } \forall v, u \in V .
\end{gathered}
$$


For a nonzero vector $\omega^{\prime} \in \operatorname{Sc}\left(V, \omega^{V}\right)$, we know that $\rho\left(\omega^{\prime}\right) \in \operatorname{Sc}\left(W, \omega^{W}\right)$. If $\operatorname{Ker} \rho \cap$ $\operatorname{Sc}\left(V, \omega^{V}\right)=\{0\}$, then $\rho\left(\omega^{\prime}\right) \neq 0$. Hence $\widehat{\rho}$ is a injective map.

For $\omega^{\prime \prime} \in \operatorname{Sc}\left(W, \omega^{W}\right)$, there is a semi-conformal subalgebra $\left(U, \omega^{\prime \prime}\right)$ of $W$ such that $\omega_{n}^{\prime \prime}=\omega_{n}^{W}$ on $U$ for $n \geq 0$. Since $\rho$ is a surjection, there exists a preimage $\omega^{\prime}$ in $V$ such that

$$
\rho\left(\omega_{n}^{\prime} \omega^{\prime}\right)=\omega_{n}^{\prime \prime} \omega^{\prime \prime}=\omega_{n}^{W} \omega^{\prime \prime}=\rho\left(\omega_{n}^{V} \omega^{\prime}\right), \text { for } n \geq 0 .
$$

It follows that $\omega_{1}^{\prime} \omega^{\prime}=\omega_{1}^{V} \omega^{\prime}=2 \omega^{\prime}$. Because of $\operatorname{Ker} \rho \cap\left(V_{1} \oplus V_{2}\right)=\{0\}$ and the relations (2.3), then we have $\omega_{n}^{\prime} \omega^{\prime}=\omega_{n}^{V} \omega^{\prime}$ for $n \geq 2$. By Remark 2.2, we also have $\omega_{0}^{\prime} \omega^{\prime}=\omega_{0}^{V} \omega^{\prime}$. Thus, by [CL, Proposition 2.2], we know that the preimage $\omega^{\prime}$ of $\omega^{\prime \prime} \in \operatorname{Sc}\left(W, \omega^{W}\right)$ is a semi-conformal vector of $\left(V, \omega^{V}\right)$. Finally, we associate the conclusion 1$)$ to get that $\widehat{\rho}$ is an isomorphism from $\operatorname{Sc}\left(V, \omega^{V}\right)$ to $\operatorname{Sc}\left(W, \omega^{W}\right)$.

2.6. In fact, the commutant of $\left(W, \omega^{W}\right)$ can induce an involution $\omega^{W}-$ of $\operatorname{Sc}\left(W, \omega^{W}\right)$ as follows

$$
\begin{aligned}
\omega^{W}-: \operatorname{Sc}\left(W, \omega^{W}\right) & \longrightarrow \operatorname{Sc}\left(W, \omega^{W}\right) \\
\omega^{\prime} & \longmapsto \omega^{W}-\omega^{\prime} .
\end{aligned}
$$

so for $\omega^{1}, \omega^{2} \in \operatorname{Sc}\left(W, \omega^{W}\right)$, we know $\omega^{W}-\omega^{1}$ and $\omega^{W}-\omega^{2}$ are conformal vectors of commutants $C_{W}\left(\left\langle\omega^{1}\right\rangle\right)$ and $C_{W}\left(\left\langle\omega^{2}\right\rangle\right)$, respectively. If $\omega^{1} \preceq \omega^{2}$, then $\omega^{W}-\omega^{2} \preceq \omega^{W}-\omega^{1}$.

\section{SEMI-CONFORMal VeCtors of AFFine VERTEX operator Algebras}

For a finite dimensional complex simple Lie algebra $\mathfrak{g}$, the corresponding (untwisted) affine Lie algebra is $\widehat{\mathfrak{g}}=\mathfrak{g} \otimes \mathbb{C}\left[t, t^{-1}\right] \oplus \mathbb{C} K$. Let $\widehat{\mathfrak{g}}_{(\geq 0)}=\mathfrak{g} \otimes \mathbb{C}[t]+\mathbb{C} K$ be the Lie subalgebra and

$$
V_{\widehat{\mathfrak{g}}}(\ell, 0)=U(\mathfrak{g}) \otimes_{U\left(\widehat{\mathfrak{g}}_{(\geq 0)}\right)} \mathbb{C}_{\ell}
$$

be the induced $\widehat{\mathfrak{g}}$-module and $L_{\widehat{\mathfrak{g}}}(\ell, 0)$ is the unique irreducible quotient of $V_{\widehat{\mathfrak{g}}}(\ell, 0)$ for all $\ell \in \mathbb{C}$. When $\ell$ is not the critical (i.e., $\ell$ is not negative of the dual Coexter number $h^{\vee}$ of $\mathfrak{g})$, then $V_{\widehat{\mathfrak{g}}}(\ell, 0)$ is a vertex operator algebra and $L_{\widehat{\mathfrak{g}}}(\ell, 0)$ is a simple vertex operator algebra (cf. [LL, 6.2]). We briefly recall the constructions for later use.

The PBW theorem implies that $V_{\widehat{\mathfrak{g}}}(\ell, 0)$ has a $\mathbb{C}$-linear basis

$$
\left\{a^{i_{1}}\left(-n_{1}\right) \cdots a^{i_{r}}\left(-n_{r}\right) \mathbf{1} \mid 1 \leq i_{1} \leq i_{2} \leq \cdots \leq i_{r} \leq d, n_{1} \geq 1, \cdots, n_{r} \geq 1\right\}
$$

if $\left\{a^{1}, \cdots, a^{d}\right\}$ is a basis of $\mathfrak{g}$. In particular,

$$
V_{n}=\mathbb{C}-\operatorname{Span}\left\{a^{i_{1}}\left(-n_{1}\right) \cdots a^{i_{r}}\left(-n_{r}\right) \mathbf{1} \mid \sum_{i=1}^{d} n_{i}=n\right\} .
$$


Hence $V_{1}=\mathfrak{g}(-1) \mathbf{1}=\mathfrak{g}$ and $V_{2}$ has a basis

$$
\left\{a^{i}(-1) a^{j}(-1) \mathbf{1} \mid 1 \leq i \leq j \leq d\right\} \cup\left\{a^{i}(-2) \mathbf{1} \mid i=1, \cdots, d\right\} .
$$

Hence $\operatorname{dim} V_{2}=d(d+3) / 2$.

Note that conformal vector $\omega$ is of $V=V_{\widehat{\mathfrak{g}}}(\ell, 0)$ is

$$
\omega=\frac{1}{2\left(\ell+h^{\vee}\right)} \sum_{i=1}^{d} u^{i}(-1) u^{i}(-1) \mathbf{1} \in V_{2}
$$

for any fixed orthogonal normal basis $\left\{u^{1}, \cdots, u^{d}\right\}$ of $\mathfrak{g}$ with respect to the normalized Killing form $\langle\cdot, \cdot\rangle$ on $\mathfrak{g}$.

Let $G$ be the adjoint algebraic group corresponding to $\mathfrak{g}$. $G$ acts on $\mathfrak{g}$ as automorphism group of the Lie algebra $\mathfrak{g}$, and thus preserving the Killing form on $\mathfrak{g}$. Since $G$ is a simple algebraic group, this action defines an embedding of $G \subset \mathrm{SO}_{\operatorname{dim} \mathfrak{g}}(\mathbb{C})$. In case that $\mathfrak{g}=\mathfrak{s l}_{2}$, we have $G=\mathrm{SO}_{3}(\mathbb{C})$ (both of type $\mathrm{A}_{1}=\mathrm{B}_{1}$ ). This is not true for higher rank Lie algebras. Thus $G$ also acts on $\widehat{\mathfrak{g}}$ as Lie algebra automorphism preserving the Lie subalgebra $\widehat{\mathfrak{g}}_{\geq 0}$ and the central element $K$. Hence $G$ acts on $\widehat{\mathfrak{g}}$-module $V_{\widehat{\mathfrak{g}}}(\ell, 0)$ compatible with the action on $\widehat{\mathfrak{g}}$, i.e., the Lie algebra module structure

$$
\widehat{\mathfrak{g}} \otimes V_{\widehat{\mathfrak{g}}}(\ell, 0) \rightarrow V_{\widehat{\mathfrak{g}}}(\ell, 0)
$$

is a $G$-module homomorphism. The $G$-action on $V_{\widehat{\mathfrak{g}}}(\ell, 0)$ can be directly written by

$$
g\left(a^{i_{1}}\left(-n_{1}\right) \cdots a^{i_{r}}\left(-n_{r}\right) \mathbf{1}\right)=g\left(a^{i_{1}}\right)\left(-n_{1}\right) \cdots g\left(a^{i_{r}}\right)\left(-n_{r}\right) \mathbf{1} .
$$

Thus $G$ also acts on $V_{\widehat{\mathfrak{g}}}(\ell, 0)$ as automorphisms of the vertex operator algebra preserving the conformal vector $\omega$. Since $L_{\widehat{\mathfrak{g}}}(\ell, 0)$ is the unique $\widehat{\mathfrak{g}}$-irreducible quotient of $V_{\widehat{\mathfrak{g}}}(\ell, 0)$, $G$ also acts on $L_{\widehat{\mathfrak{g}}}(\ell, 0)$. Let $\pi: V_{\widehat{\mathfrak{g}}}(\ell, 0) \rightarrow L_{\widehat{\mathfrak{g}}}(\ell, 0)$ be the quotient map. Thus $\pi$ is a conformal homomorphism of vertex operator algebras, i.e., $\pi(\omega)$ is the conformal vector of $L_{\widehat{\mathfrak{g}}}(\ell, 0)$. Then $\pi$ is a $G$-module homomorphism.

In particular, the group $G$ acts on $\operatorname{Sc}\left(V_{\widehat{\mathfrak{g}}}(\ell, 0)\right), \operatorname{Sc}\left(L_{\widehat{\mathfrak{g}}}(\ell, 0)\right), \operatorname{ScAlg}\left(V_{\widehat{\mathfrak{g}}}(\ell, 0)\right)$, and $\operatorname{ScAlg}\left(L_{\widehat{\mathfrak{g}}}(\ell, 0)\right)$ such that the following diagram commutes with all maps being $G$-equivariant

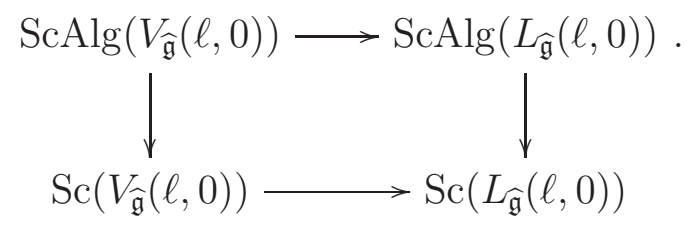

We are interested in computing $G$-orbit structures of the above varieties. And we will first focus on $V=V_{\widehat{\mathfrak{g}}}(\ell, 0)$. Note that $V_{1}=\mathfrak{g}$ as a Lie algebra and $V_{n}$ is a module over the 
Lie algebra $V_{1}$ under the action $a \cdot v=a_{0}(v)$ for all $v \in V_{n}$. Recall that $a(n)=a \otimes t^{n} \in \widehat{\mathfrak{g}}$. We will repeatedly use the following formulas [LL, (6.2.67)]

$$
\begin{gathered}
a(n) b(m)=b(m) a(n)+[a, b](n+m)+n \delta_{n+m, 0}\langle a, b\rangle K \\
{[L(n), \beta(m)]=-m \beta(m+n), \text { for all } \beta \in \mathfrak{g}, \text { for all } m, n \in \mathbb{Z} .}
\end{gathered}
$$

Since $[L(n), a(0)]=0$ for all $a \in \mathfrak{g}$, each $L(n): V_{m} \rightarrow V_{m-n}$ is a $\mathfrak{g}$-module homomorphism. And since $L(1) a(-2) \mathbf{1}=2 a(-1) \mathbf{1}$. Thus $L(1): V_{2} \rightarrow V_{1}$ is a surjective $\mathfrak{g}$-module homomorphism. Thus in $V_{2}$, the vector subspace spanned by $\left\{u^{i}(-2) \mathbf{1} \mid 1 \leq i \leq \operatorname{dim} \mathfrak{g}\right\}$ as a $\mathfrak{g}$-module is isomorphic to $\mathfrak{g}$ and the quotient of $V_{2}$ by this submodule is a $\mathfrak{g}$-module isomorphic to the second symmetric power $\operatorname{Sym}^{2}(\mathfrak{g})$. Since $V_{2}$ is finite dimensional, thus we have $V_{2}$ isomorphic to $\mathfrak{g} \oplus \operatorname{Sym}^{2}(\mathfrak{g})$ as a $\mathfrak{g}$-module and $\operatorname{Ker} L(1) \cap V_{2}$ is a $\mathfrak{g}$-submodule. Thus the set of all semi-conformal vectors are solutions of the equaltion $L^{\prime}(0) \omega^{\prime}=2 \omega^{\prime}$.

Choose an orthonormal basis $\left\{u^{1}, \cdots, u^{d}\right\}$ of $\mathfrak{g}$ with respect to the Killing form and we define the structure constants $R=\left(\gamma_{i j}^{s}\right)$ with respect to this basis by

$$
\left[u^{i}, u^{j}\right]=\sum_{s=1}^{d} \gamma_{i j}^{s} u^{s} .
$$

Then for each fixed $s, R^{s}$ is a skew symmetric matrix. We will use the following conventions

(1) For each pair $(j, s), R_{* j}^{s}=\left[\gamma_{1 j}^{s}, \gamma_{2 j}^{s}, \cdots, \gamma_{d j}^{s}\right]$ is a row vector;

(2) For each pair $(j, k), R_{k *}^{j}=\left[\gamma_{k 1}^{j}, \gamma_{k 2}^{j}, \cdots, \gamma_{k d}^{j}\right]^{\text {tr }}$ is a column vector;

(3) For each $m, R^{m}=\left(\gamma_{i j}^{m}\right)$ is a matrix and $R_{m *}^{*}=\left(\gamma_{m i}^{j}\right)$ is a matrix with the $(i, j)$ entry being $\gamma_{m i}^{j}$;

(4) For a matrix $M$, we denoted the $j$-th column by $M_{j}$ and the $j$-th row by $M^{j}$ and $M_{i j}$ for the $(i, j)$-entry of $M$;

(5) Let $A$ be the matrix with the $(i, j)$ entry being $a_{i j}$. For each $i, A R^{i}$ is a matrix with the $(r, s)$ entry being $\left(A R^{i}\right)_{r s}=\sum_{h=1}^{d} a_{r h} \gamma_{h s}^{i}$;

(6) Let $A$ be the matrix with the $(i, j)$ entry being $a_{i j}$. We denote a matrix with the $(i, j)$ entry being $\sum_{r, s=1}^{d}\left(A R^{i}\right)_{r s}\left(A R^{j}\right)_{s r}$ by $\left(\sum_{r, s=1}^{d}\left(A R^{i}\right)_{r s}\left(A R^{j}\right)_{s r}\right)$

(7) Let $A$ be the matrix with the $(i, j)$ entry being $a_{i j}$. We denote the matrix with the $(i, j)$ entry being $\sum_{r, s=1}^{d}\left(A R^{s}\right)_{i r}\left(A R^{j}\right)_{r s}$ by $\left(\sum_{r, s=1}^{d}\left(A R^{s}\right)_{i r}\left(A R^{j}\right)_{r s}\right)$.

We use $\mathbf{u}(-1)=\left[u^{1}(-1), \cdots, u^{d}(-1)\right]$ to denote an ordered basis of $\mathfrak{g} \otimes t^{-1} \subseteq \widehat{\mathfrak{g}}$. In the following proposition, we give an explicit description of the $\mathfrak{g}$-submodule $\operatorname{Ker} L(1) \cap V_{\widehat{\mathfrak{g}}}(\ell, 0)_{2}$. 
Proposition 3.1. Elements $\omega^{\prime} \in \operatorname{Ker} L(1) \bigcap V_{\widehat{\mathfrak{g}}}(\ell, 0)_{2}$ correspondes to a unique symmetric $d \times d$-matrix $A$ such that $\omega^{\prime}=\mathbf{u}(-1) A \mathbf{u}(-1)^{t r} \mathbf{1}$. Or equivalently, $\operatorname{Ker} L(1) \cap V_{\widehat{\mathfrak{g}}}(\ell, 0)_{2}$ isomomorphic to the space of all quadratic forms on $\mathfrak{g}^{*}$ as $G$-modules.

Proof. We can write $\omega^{\prime} \in V_{\widehat{\mathfrak{g}}}(\ell, 0)_{2}$ as

$$
\omega^{\prime}=\sum_{i, j=1}^{d} a_{i j} u^{i}(-1) u^{j}(-1) \mathbf{1}+\sum_{s=1}^{d} b_{s} u^{s}(-2) \mathbf{1}
$$

Using $L(1) \mathbf{1}=u^{i}(0) \mathbf{1}=0$, we have

$$
\begin{aligned}
L(1) \omega^{\prime} & =\sum_{i, j=1}^{n} a_{i j} L(1) u^{i}(-1) u^{j}(-1) \mathbf{1}+\sum_{s=1}^{n} b_{s} L(1) u^{s}(-2) \mathbf{1} \\
& =\sum_{i, j=1}^{n} a_{i j}\left[L(1), u^{i}(-1)\right] u^{j}(-1) \mathbf{1}+\sum_{i, j=1}^{n} a_{i j} u^{i}(-1)\left[L(1), u^{j}(-1)\right] \mathbf{1}+\sum_{s=1}^{n} b_{s}\left[L(1), u^{s}(-2)\right] \mathbf{1} \\
& =\sum_{i, j=1}^{n} a_{i j} u^{i}(0) u^{j}(-1) \mathbf{1}+\sum_{i, j=1}^{n} a_{i j} u^{i}(-1) u^{j}(0) \mathbf{1}+2 \sum_{s=1}^{n} b_{s} u^{s}(-1) \mathbf{1} \\
& =\sum_{i, j=1}^{n} a_{i j}\left[u^{i}(0), u^{j}(-1)\right] \mathbf{1}+\sum_{i, j=1}^{n} a_{i j} u^{j}(-1) u^{i}(0) \mathbf{1}+2 \sum_{s=1}^{n} b_{s} u^{s}(-1) \mathbf{1} \\
& =\sum_{i, j=1}^{n} a_{i j}\left[u^{i}(0), u^{j}(-1)\right] \mathbf{1}+2 \sum_{s=1}^{n} b_{s} u^{s}(-1) \mathbf{1} \\
& =\sum_{i, j, l}^{n} a_{i j} \gamma_{i j}^{l} u^{l}(-1) \mathbf{1}+2 \sum_{s=1}^{n} b_{s} u^{s}(-1) \mathbf{1} \\
& =\sum_{l=1}^{n}\left(\sum_{i, j}^{n} a_{i j} \gamma_{i j}^{l}+2 b_{l}\right) u^{l}(-1) \mathbf{1} \\
& =0 .
\end{aligned}
$$

This implies $\sum_{i, j=1}^{d} a_{i j} \gamma_{i j}^{l}+2 b_{l}=0$. Noting that $\gamma_{i i}^{l}=0$ and $\gamma_{i j}^{l}=-\gamma_{j i}^{l}$, we have

$$
\begin{aligned}
b_{l} & =-\frac{1}{2} \sum_{i, j=1}^{d} a_{i j} \gamma_{i j}^{l}=-\frac{1}{2}\left(\sum_{1 \leq i<j \leq d} a_{i j} \gamma_{i j}^{l}+\sum_{d \geqq i>j \geqq 1}^{n} a_{i j} \gamma_{i j}^{l}\right) \\
& =-\frac{1}{2} \sum_{1 \leq i<j \leq d}\left(a_{i j}-a_{j i}\right) \gamma_{i j}^{l} .
\end{aligned}
$$

If the matrix $A=\left(a_{i j}\right)$ is symmetric, then $\mathbf{u}(-1) A \mathbf{u}(-1)^{\operatorname{tr}} \mathbf{1} \in \operatorname{ker}(L(1))$.

The assignment $A \mapsto \mathbf{u}(-1) A \mathbf{u}(-1)^{t r} \mathbf{1} \in \operatorname{ker}(L(1))$ defines a linear map from the space of quadratic forms $\operatorname{Sym}^{2}(\mathfrak{g})$ to $\operatorname{ker}(L(1)) \cap V_{2}$, which is injective. Comparing their dimensions give an isomorphism. Note that $L(1): V_{2} \rightarrow V_{1} \cong \mathfrak{g}(-1) \mathbf{1}$ is surjective.

Note that $\operatorname{Sym}^{2}(\mathfrak{g})=\mathbb{C}\left[\mathfrak{g}^{*}\right]_{2}$ is the space of all degree two symmetric polynomial functions on the dual vector space $\mathfrak{g}^{*}$. 
From now on we can write elements in $\operatorname{ker}(L(1)) \cap V_{2}$ in terms of $\omega^{\prime}=\mathbf{u}(-1) A \mathbf{u}(-1)^{\operatorname{tr}} \mathbf{1}$ with $A$ being a symmetric matrix and write $Y\left(\omega^{\prime}, z\right)=\sum_{n \in \mathbb{Z}} L^{\prime}(n) z^{-n-2}$. Similar to the computation of Proposition 3.1, we can obtain the following equations in Proposition $3.2-3.4$.

Proposition 3.2. For $\omega^{\prime}=\mathbf{u}(-1) A \mathbf{u}(-1)^{t r} \mathbf{1}, L^{\prime}(1) \omega^{\prime}=0$ if and only if

$$
\sum_{s=1}^{d} \sum_{j=1}^{d}\left(R_{* j}^{s}\left[\sum_{l=1}^{d}\left(A R^{l} A\right) R_{l *}^{j}+\ell\left(A^{2}\right)_{j}\right]\right) u^{s}(-1) \mathbf{1}=0 .
$$

Proposition 3.3. The vector $\omega^{\prime}=\mathbf{u}(-1) A \mathbf{u}(-1)^{t r} \mathbf{1}$ satisfies $L^{\prime}(0) \omega^{\prime}=2 \omega^{\prime}$ if and only if

$$
\left.\left.2 \ell A^{2}+\sum_{m=1}^{d} A R^{m} A R_{m *}^{*}+\left(\sum_{r, s=1}^{d}\left(A R^{i}\right)_{r s}\left(A R^{j}\right)_{s r}\right)\right)+\left(\sum_{r, s=1}^{d}\left(A R^{s}\right)_{i r}\left(A R^{j}\right)_{s r}\right)\right)=A
$$

and

$$
\ell A\left(\sum_{i=1}^{d} R^{i} A_{i}\right)=0
$$

Proposition 3.4. The vector $\omega^{\prime}=\mathbf{u}(-1) A \mathbf{u}(-1)^{\operatorname{tr}} \mathbf{1}$ satisfies $L(2) \omega^{\prime}=L^{\prime}(2) \omega^{\prime}=\frac{c^{\prime}}{2} \mathbf{1}$ if and only if the symmetric $A=\left(a_{i j}\right)$ satisfies the following equations

$$
\begin{gathered}
c^{\prime}=2 \ell \operatorname{tr}(A) \\
\ell \operatorname{tr}(A)=2 \ell^{2} \operatorname{tr}\left(A^{2}\right)+2 \ell \sum_{i, j=1}^{d}\left((A R)^{j}(A R)^{i}\right)_{(i, j)}-\ell \sum_{i=1}^{d} \operatorname{tr}\left(A^{2}\left(R^{i}\right)^{2}\right) .
\end{gathered}
$$

Therefore we have

Theorem 3.5. A vector $\omega^{\prime}=\mathbf{u}(-1) A \mathbf{u}(-1)^{\operatorname{tr}} \mathbf{1} \in \operatorname{Sc}\left(V_{\widehat{\mathfrak{g}}}(\ell, 0), \omega\right)$ if and only if the symmetric matrix $A=\left(a_{i j}\right)$ satisfies equations (3.4)-(3.8).

Remark 3.6. If $\omega^{\prime} \neq 0$ is in $\operatorname{Sc}\left(V_{\widehat{\mathfrak{g}}}(\ell, 0), \omega\right)$, then for $\alpha \in \mathbb{C}, \alpha \omega^{\prime} \in \operatorname{Sc}\left(V_{\widehat{\mathfrak{g}}}(\ell, 0), \omega\right)$ if and only if $\alpha=0,1$. This follows from (3.5).

Remark 3.7. Here, we can generalize to more general cases of vertex operator algebra $(V, \omega)$. Let $(V, \omega)$ be a CFT-type vertex operator algebra ([DLMM, DM]) with the assumption that $V_{2}$ (the subspace with degree 2 of $V$ ) is generated by $V_{1}$ (the subspace with degree 1 of $V)$. It is well-known that $V_{1}$ is a Lie algebra with the Lie bracket $[u, v]=u_{0} v$ for $u, v \in V_{1}$ and a bilinear form $\langle u, v\rangle \mathbf{1}=u_{1} v$ for $u, v \in V_{1}$. At first, we choose a basis of the Lie algebra $V_{1}$. Then a vector belongs to $\operatorname{Ker} L(1) \cap V_{2}$ if and only if there exists a symmetric matrix such that it can be also expressed as a quadratic form with respect to the fixed basis of $V_{1}$. This proposition is a key for us to compute the sufficient and 
necessary condition semi-conformal vectors of $V$ satisfy. With the help of this proposition, we also obtain the matrix equations of semi-conformal vectors of $V$ satisfy as similar as the equations in Theorem 3.5 .

The automorphism group $\operatorname{Aut}(\mathfrak{g})$ acts on the set $\operatorname{Sc}\left(V_{\widehat{\mathfrak{g}}}(\ell, 0), \omega\right)$ is given by matrix congruence, i.e., if $\sigma \in \operatorname{Aut}(\mathfrak{g})$ has matrix $g=\left(g_{i j}\right)$. Then $\sigma(\omega)$ corresponds to the symmetric matrix $g A g^{t r}$. This follows from

$$
\sigma\left(\mathbf{u}(-1) A \mathbf{u}(-1)^{t r} \mathbf{1}\right)=\sigma(\mathbf{u}(-1)) A \sigma\left(\mathbf{u}(-1)^{t r}\right) \mathbf{1}=(\mathbf{u}(-1) g) A\left(g^{t r} \mathbf{u}(-1)^{t r}\right) \mathbf{1} .
$$

Note that $g R^{s} g^{t r}=R^{s}$ for all $s$, and the matrix $g \in \mathrm{SO}_{d}(\mathfrak{g})$. Thus each of the equations (3.4) - (3.8) are invariant under $g$. Therefore the question becomes a question purely on symmetric matrices. Under the congruence action by the group $\operatorname{Aut}(\mathfrak{g})$. Thus the question is to classify the $\operatorname{Aut}(\mathfrak{g})$-orbits in the set of symmetric matrices satisfy the equations (3.4)(3.8).

According to Proposition 3.1, for each $\omega^{\prime} \in \operatorname{Sc}\left(V_{\widehat{\mathfrak{s l}}_{2}}(\ell, 0), \omega\right)$, if $A$ is the corresponding symmetric matrix, then we can denote by $\omega_{A}=\omega^{\prime}$. From the above statements, we have

Proposition 3.8. The action of $\operatorname{Aut}(\mathfrak{g})$ on $\operatorname{Sc}\left(V_{\widehat{\mathfrak{g}}}(\ell, 0), \omega\right)$ is defined as follows: Fixing an orthonormal basis $\left\{u^{1}, \cdots, u^{d}\right\}$ of $\mathfrak{g}$, for each $\omega_{A} \in \operatorname{Sc}\left(V_{\widehat{\mathfrak{g}}}(\ell, 0), \omega\right)$ and $\forall \sigma \in \operatorname{Aut}(\mathfrak{g})$, we have $\sigma\left(\omega_{A}\right)=\omega_{B} \in \operatorname{Sc}\left(V_{\widehat{\mathfrak{g}}}(\ell, 0), \omega\right)$, where $B=g A g^{\text {tr }}$ and $g$ is the matrix of $\sigma$ with respect to the basis $\left\{u^{1}, \cdots, u^{d}\right\}$.

Example 3.9. Here we give some examples of semi-conformal vectors of $\left(V_{\widehat{\mathfrak{s}}_{2}}(\ell, 0), \omega\right)$. Of course, $\omega$ is a semi-conformal vector with the corresponding symmetric matrix

$$
A=\left(\begin{array}{ccc}
\frac{1}{2(\ell+2)} & 0 & 0 \\
0 & \frac{1}{2(\ell+2)} & 0 \\
0 & 0 & \frac{1}{2(\ell+2)}
\end{array}\right)
$$

Let $\mathfrak{h}=\mathbb{C} u^{3}$. It generates a Heisenberg vertex operator algebra $V_{\widehat{\eta}}(\ell, 0)$ in $\left(V_{\widehat{\mathfrak{s l}}_{2}}(\ell, 0), \omega\right)$ with the conformal vector $\omega_{\mathfrak{h}}=\frac{1}{2 \ell} u^{3}(-1)^{2} \mathbf{1} \in \operatorname{Sc}\left(V_{\widehat{\mathfrak{s l}}_{2}}(\ell, 0), \omega\right)$. Its corresponding symmetric matrix is

$$
A_{\mathfrak{h}}=\left(\begin{array}{ccc}
0 & 0 & 0 \\
0 & 0 & 0 \\
0 & 0 & \frac{1}{2 \ell}
\end{array}\right) .
$$

The commutant of $V_{\widehat{\mathfrak{h}}}(\ell, 0)$ in $\left(V_{\widehat{\mathfrak{s l}}_{2}}(\ell, 0), \omega\right)$ is well-known as a parafemion vertex operator algebra(cf. [DLY, DW1, DW2]), and its corresponding symmetric matrix is $A-A_{\mathfrak{h}}$. 
Denote by

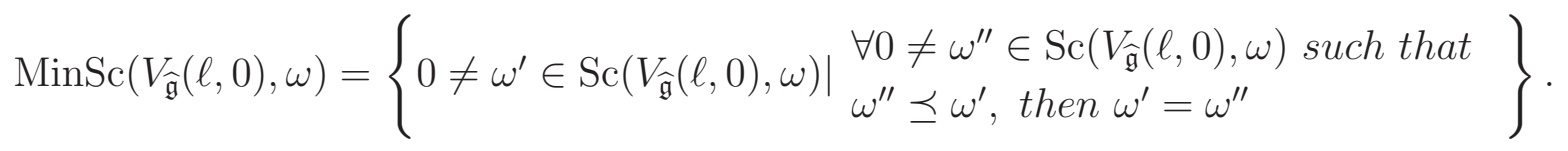

Proposition 3.10. Let $(V, \omega)$ be a vertex operator algebra with conformal vector $\omega$. For for any $\omega^{\prime}, \omega^{\prime \prime} \in \operatorname{Sc}(V, \omega)$ we have $\omega^{\prime} \preceq \omega^{\prime \prime}$ if and only if $\sigma\left(\omega^{\prime}\right) \preceq \sigma\left(\omega^{\prime \prime}\right)$ for each $\sigma \in$ $\operatorname{Aut}(V, \omega)$. In particular, if $\omega^{\prime} \in \operatorname{Min} \operatorname{Sc}(V, \omega)$, then $\sigma\left(\omega^{\prime}\right) \in \operatorname{Min} \operatorname{Sc}(V, \omega)$ for each $\sigma \in$ $\operatorname{Aut}(V, \omega)$.

Proof. For any $\sigma \in \operatorname{Aut}(V)$, we have

$$
\left[Y\left(u, z_{1}\right), Y\left(v, z_{2}\right)\right]=0 \text { if and only if }\left[Y\left(\sigma(u), z_{1}\right), Y\left(\sigma(v), z_{2}\right)\right]=0
$$

for all $u, v \in V$. Therefore,

$$
\sigma\left(C_{V}(U)\right)=C_{V}(\sigma(U))
$$

for any vertex subalgebra $U$. Hence the proposition follows by considering the vertex operator subalgebras $\left\langle\omega^{\prime}\right\rangle$ and $\left\langle\omega^{\prime \prime}\right\rangle$.

Proposition 3.11. When $\ell$ is a positive integer, we have

$$
\operatorname{Sc}\left(V_{\widehat{\mathfrak{g}}}(\ell, 0), \omega\right)=\operatorname{Sc}\left(L_{\widehat{\mathfrak{g}}}(\ell, 0), \omega\right) .
$$

Proof. When $\ell$ is a positive integer, $L_{\widehat{\mathfrak{g}}}(\ell, 0)=V_{\widehat{\mathfrak{g}}}(\ell, 0) /<e_{\theta}^{\ell+1}(-1) \mathbf{1}>$, where $\theta$ is the highest root of $\mathfrak{g}$. If $\ell \geq 2$, then we note that $\operatorname{Sc}\left(V_{\widehat{\mathfrak{g}}}(\ell, 0), \omega\right) \cap<e_{\theta}^{\ell+1}(-1) \mathbf{1}>=\{0\}$. By Lemma 2.4. 2), we have $\operatorname{Sc}\left(V_{\widehat{\mathfrak{g}}}(\ell, 0), \omega\right) \hookrightarrow \operatorname{Sc}\left(L_{\widehat{\mathfrak{g}}}(\ell, 0), \omega\right)$. Actually, since $V_{\widehat{\mathfrak{g}}}(\ell, 0)_{2} \cap<$ $e_{\theta}^{\ell+1}(-1) \mathbf{1}>=\{0\}$, then $V_{\widehat{\mathfrak{g}}}(\ell, 0)_{2}=L_{\widehat{\mathfrak{g}}}(\ell, 0)_{2}$, so we have $\operatorname{Sc}\left(V_{\widehat{\mathfrak{g}}}(\ell, 0), \omega\right)=\operatorname{Sc}\left(L_{\widehat{\mathfrak{g}}}(\ell, 0), \omega\right)$. If $\left.\ell=1, V_{\widehat{\mathfrak{g}}}(\ell, 0)_{2} \cap<e_{\theta}^{2}(-1) \mathbf{1}\right\rangle=\mathbb{C} e_{\theta}^{2}(-1) \mathbf{1}$. Obviously, $e_{\theta}^{2}(-1) \mathbf{1} \notin \operatorname{Sc}\left(V_{\widehat{\mathfrak{g}}}(\ell, 0), \omega\right)$, then $\operatorname{Sc}\left(V_{\widehat{\mathfrak{g}}}(\ell, 0), \omega\right) \cap<e_{\theta}^{\ell+1}(-1) \mathbf{1}>=\{0\}$. By Lemma 2.4. 2), we have $\operatorname{Sc}\left(V_{\widehat{\mathfrak{g}}}(\ell, 0), \omega\right) \hookrightarrow$ $\operatorname{Sc}\left(L_{\widehat{\mathfrak{g}}}(\ell, 0), \omega\right)$. Since $e_{\theta}^{2}(-1) \mathbf{1} \notin \operatorname{Sc}\left(V_{\widehat{\mathfrak{g}}}(\ell, 0), \omega\right)$, then $\operatorname{Sc}\left(V_{\widehat{\mathfrak{g}}}(\ell, 0), \omega\right)=\operatorname{Sc}\left(L_{\widehat{\mathfrak{g}}}(\ell, 0), \omega\right)$.

\section{CASE $A_{1}^{(1)}$}

4.1. Set $\mathfrak{g}=\mathfrak{s l}_{2}$. Then the corresponding group $G=\mathrm{PSL}_{2}(\mathbb{C})=\mathrm{SL}_{2}(\mathbb{C}) /\{ \pm I\}$ acts on $\mathfrak{g}$ by conjugation of matrices. Let $h, e, f$ be a standard Chevalley basis of $\mathfrak{s l}_{2}$. The normalized Killing form $\langle\cdot, \cdot\rangle$ on $\mathfrak{g}$ with respect to this Chevalley basis has the form

$$
\langle h, h\rangle=2,\langle e, f\rangle=1,\langle h, e\rangle=\langle h, f\rangle=\langle e, e\rangle=\langle f, f\rangle=0 .
$$

The dual Coexter number $h^{V}=2$ (the notation $h^{V}$ will not cause confusion in this section since we will simply use 2 for the dual Coexter number leaving $h$ for the standard basis 
for $\left.\mathfrak{s l}_{2}\right)$. Thus the conformal vector $\omega$ of $V_{\widehat{\mathfrak{g}}}(\ell, 0)$ is

$$
\begin{gathered}
\omega=\frac{1}{2(\ell+2)}\left(\frac{1}{2} h(-1)^{2} \mathbf{1}+e(-1) f(-1) \mathbf{1}+f(-1) e(-1) \mathbf{1}\right) \\
=\frac{1}{2(\ell+2)}\left(\frac{1}{2} h(-1)^{2} \mathbf{1}+2 e(-1) f(-1) \mathbf{1}-h(-2) \mathbf{1}\right) .
\end{gathered}
$$

The central charge of $V=V_{\widehat{\mathfrak{g}}}(\ell, 0)$ is $3 \ell /(\ell+2)$. Let $\mathcal{J} \subseteq V_{\widehat{\mathfrak{g}}}(\ell, 0)$ be the unique maximal $\widehat{\mathfrak{g}}$-submodule such that $L_{\widehat{\mathfrak{g}}}(\ell, 0)=V_{\widehat{\mathfrak{g}}}(\ell, 0) / \mathcal{J}$. Note that when $\ell \in \mathbb{Z}_{\geq 0}, \mathcal{J}$ is the $\widehat{\mathfrak{g}}$ submodule generated by $e(-1)^{\ell+1} \mathbf{1}$. In particular, when $\ell>1$, then $\mathcal{J} \subseteq \oplus_{n>2} V_{n}$.

We can give an orthonormal basis with respect to the normalized Killing form on $\mathfrak{s l}_{2}$ :

$$
\alpha_{1}=\frac{e+f}{\sqrt{2}}=\frac{1}{\sqrt{2}}\left(\begin{array}{ll}
0 & 1 \\
1 & 0
\end{array}\right) ; \alpha_{2}=\frac{e-f}{\sqrt{-2}}=\frac{1}{\sqrt{2}}\left(\begin{array}{cc}
0 & -\mathbf{i} \\
\mathbf{i} & 0
\end{array}\right) ; \alpha_{3}=\frac{h}{\sqrt{2}}=\frac{1}{\sqrt{2}}\left(\begin{array}{cc}
1 & 0 \\
0 & -1
\end{array}\right) \text {. }
$$

The structure constants $R=\left(\gamma_{i j}^{s}\right)$ is given in the following

$$
\left[\alpha_{1}, \alpha_{2}\right]=\sqrt{2} \mathbf{i} \alpha_{3} ;\left[\alpha_{2}, \alpha_{3}\right]=\sqrt{2} \mathbf{i} \alpha_{1} ;\left[\alpha_{3}, \alpha_{1}\right]=\sqrt{2} \mathbf{i} \alpha_{2}
$$

Thus $\gamma_{i j}^{s}=\sqrt{2} \mathbf{i}$ if $(i j s)$ is one of the three cycles (123), (231), (312) and zero otherwise. Then the conformal vector of $L_{\widehat{\mathfrak{s l}}_{2}}(\ell, 0)$ can be rewritten as

$$
\omega=\frac{1}{2(\ell+2)} \sum_{i=1}^{3} \alpha_{i}(-1)^{2} \mathbf{1}
$$

Since $G=\mathrm{PSL}_{2}(\mathbb{C})$ action on $\mathfrak{g}$ preserves the killing form, then $\mathrm{PSL}_{2}(\mathbb{C}) \subset \mathrm{SO}_{3}(\mathbb{C})$. In fact, since $\sigma \mapsto g$ gives a covering from $\mathrm{SL}_{2}(\mathbb{C})$ to $\mathrm{SO}_{3}(\mathbb{C})$, then $\mathrm{PSL}_{2}(\mathbb{C})=\mathrm{SO}_{3}(\mathbb{C})$.

4.2. Let

$$
A=\left(\begin{array}{lll}
a_{1} & 0 & 0 \\
0 & a_{2} & 0 \\
0 & 0 & a_{3}
\end{array}\right)
$$

be a diagonal matrix. According to equations (3.4)-(3.8), we have $\omega_{A} \in \operatorname{Sc}\left(V_{\widehat{\mathfrak{s l}}_{2}}(\ell, 0), \omega\right)$ if and only if $A$ satisfies

$$
\left\{\begin{array}{l}
4\left(a_{1} a_{2}+a_{1} a_{3}-a_{2} a_{3}\right)+2 \ell a_{1}^{2}=a_{1} \\
4\left(a_{1} a_{2}+a_{2} a_{3}-a_{1} a_{3}\right)+2 \ell a_{2}^{2}=a_{2} \\
4\left(a_{1} a_{3}+a_{2} a_{3}-a_{1} a_{2}\right)+2 \ell a_{3}^{2}=a_{3} .
\end{array}\right.
$$

Theorem 4.1. When $\ell \neq-2,0,1$, we have

$$
\operatorname{Sc}\left(V_{\widehat{\mathfrak{s l}}_{2}}(\ell, 0), \omega\right)=\left\{\omega_{B} \mid B=o A o^{\text {tr }} \text { for a solution } A \text { of (4.4) and } o \in \mathrm{SO}_{3}(\mathbb{C})\right\} \text {. }
$$

Proof. For any $\omega_{B} \in \operatorname{Sc}\left(V_{\mathfrak{s l}_{2}}(\ell, 0), \omega\right)$, since $B$ is a symmetric matrix, then there exists an $o \in \mathrm{SO}_{3}(\mathbb{C})$ such that $o B o^{t r}=A$ is a diagonal matrix. According to Proposition 3.8 , 
there exists a $\sigma \in \mathrm{PSL}_{2}(\mathbb{C})$ such that $\omega_{A}=\sigma\left(\omega_{B}\right)$. Thus, $\omega_{A}$ is a semi-conformal vector of $\operatorname{Sc}\left(V_{\widehat{\mathfrak{s l}}_{2}}(\ell, 0), \omega\right)$. Hence $A$ is a solution of equations (4.4).

If $B=o A o^{t r}$ for a solution $A$ of equations (4.4) and $o \in \mathrm{SO}_{3}(\mathbb{C})$, according to Proposition 3.8, there exists a $\sigma \in \mathrm{PSL}_{2}(\mathbb{C})$ such that $\sigma\left(\omega_{A}\right)=\omega_{B}$. $\operatorname{So} \omega_{B} \in \operatorname{Sc}\left(V_{\widehat{\mathfrak{s}}_{2}}(\ell, 0), \omega\right)$.

When $\ell=0$, we solve equations (4.4) and they only have one resolution $a_{1}=a_{2}=a_{3}=$ $\frac{1}{4}$, i.e., there is only a diagonal matrix $\frac{1}{4} I$, which is just the corresponding semi-conformal vector $\omega$, so $\operatorname{Sc}\left(V_{\widehat{\mathfrak{s l}}_{2}}(\ell, 0), \omega\right)=\{0, \omega\}$; From $[\mathrm{LL}, 6.6]$, we have $L_{\widehat{\mathfrak{s l}}_{2}}(0,0)=\mathbb{C}$. Hence $\operatorname{Sc}\left(L_{\widehat{\mathfrak{s}}_{2}}(0,0), 0\right)=\{0\}$.

When $\ell=4$, we shall discuss solutions of equations (4.4) and study $\mathrm{PSL}_{2}(\mathbb{C})$-orbits of $\operatorname{Sc}\left(V_{\widehat{\mathfrak{s l}}_{2}}(4,0), \omega\right)$ and $\operatorname{Sc}\left(L_{\widehat{\mathfrak{s l}}_{2}}(4,0), \omega\right)$ in next section.

When $\ell \neq-2,0,4$, the equations (4.4) have exactly six matrix solutions $A_{1}, \cdots, A_{6}$ as follows

$$
\begin{gathered}
A_{1}=\left(\begin{array}{ccc}
\frac{1}{2 \ell} & 0 & 0 \\
0 & 0 & 0 \\
0 & 0 & 0
\end{array}\right), A_{2}=s_{1} A_{1} s_{1}^{t r}, A_{3}=s_{2} A_{1} s_{2}^{t r} ; \\
A_{4}=\left(\begin{array}{ccc}
-\frac{1}{\ell(\ell+2)} & 0 & 0 \\
0 & \frac{1}{2(\ell+2)} & 0 \\
0 & 0 & \frac{1}{2(\ell+2)}
\end{array}\right), A_{5}=s_{1} A_{4} s_{1}^{t r}, A_{6}=s_{2} A_{4} s_{2}^{t r},
\end{gathered}
$$

where

$$
s_{1}=\left(\begin{array}{rrr}
0 & 1 & 0 \\
-1 & 0 & 0 \\
0 & 0 & 1
\end{array}\right), s_{2}=\left(\begin{array}{rrr}
0 & 0 & 1 \\
0 & 1 & 0 \\
-1 & 0 & 0
\end{array}\right) \in \mathrm{SO}_{3}(\mathbb{C}) .
$$

Let $\left\{\omega_{A_{i}} \mid i=1, \cdots, 6\right\}$ be semi-conformal vectors determined by diagonal matrices which form a subset of $\operatorname{Sc}\left(V_{\widehat{\mathfrak{s l}}_{2}}(\ell, 0), \omega\right)$. Denote by

$$
\operatorname{Orb}_{A_{i}}=\left\{\omega_{B} \mid B=o A o^{\text {tr }} \text { for all } o \in \mathrm{SO}_{3}(\mathbb{C})\right\}
$$

for $i=1, \cdots, 6$, which are $\mathrm{PSL}_{2}(\mathbb{C})$-orbits of $\omega_{A_{i}}$, respectively.

Corollary 4.2. When the level $\ell \neq-2,0,1,4$,

$$
\operatorname{Sc}\left(V_{\widehat{\mathfrak{s}}_{2}}(\ell, 0), \omega\right)=\operatorname{Orb}_{A_{1}} \cup \operatorname{Orb}_{A_{4}} \cup\{0\} \cup\{\omega\} .
$$

In particular, when $\ell \in \mathbb{Z}_{+}$and $\ell \notin 1,4$,

$$
\operatorname{Sc}\left(L_{\widehat{\mathfrak{s}}_{2}}(\ell, 0), \omega\right)=\operatorname{Orb}_{A_{1}} \cup \operatorname{Orb}_{A_{4}} \cup\{0\} \cup\{\omega\} .
$$

Proof. According to Proposition 3.8, there exist a $\sigma_{1} \in \mathrm{PSL}_{2}(\mathbb{C})$ defined $\alpha_{1} \mapsto-\alpha_{2} ; \alpha_{2} \mapsto$ $\alpha_{1} ; \alpha_{3} \mapsto \alpha_{3}$ such that $\sigma_{1}\left(\omega_{A_{1}}\right)=\omega_{A_{2}}$ and $\sigma_{1}\left(\omega_{A_{4}}\right)=\omega_{A_{5}}$. As the same reason, there exist a $\sigma_{2} \in \mathrm{PSL}_{2}(\mathbb{C})$ defined $\alpha_{1} \mapsto-\alpha_{3} ; \alpha_{2} \mapsto \alpha_{2} ; \alpha_{3} \mapsto \alpha_{1}$ such that $\sigma_{1}\left(\omega_{A_{1}}\right)=\omega_{A_{3}}$ and 
$\sigma_{1}\left(\omega_{A_{4}}\right)=\omega_{A_{6}}$. So $\operatorname{Orb}_{A_{1}}, \operatorname{Orb}_{A_{2}}$ and $\operatorname{Orb}_{A_{3}}$ are the same orbit, Orb $A_{4}, \operatorname{Orb}_{A_{5}}, \mathrm{Orb}_{A_{6}}$ are the same orbit. Thus, we can get desired $\operatorname{Sc}\left(V_{\widehat{\mathfrak{s l}}_{2}}(\ell, 0), \omega\right)=\operatorname{Orb}_{A_{1}} \cup \mathrm{Orb}_{A_{4}} \cup\{0\} \cup\{\omega\}$. By Proposition 3.11, when $\ell \in \mathbb{Z}_{+}$and $\ell \notin 1,4$, we have also

$$
\operatorname{Sc}\left(L_{\widehat{\mathfrak{s l}}_{2}}(\ell, 0), \omega\right)=\operatorname{Orb}_{A_{1}} \cup \operatorname{Orb}_{A_{4}} \cup\{0\} \cup\{\omega\} .
$$

Proposition 4.3. If $\ell=1$, we have

$$
\operatorname{Sc}\left(V_{\widehat{\mathfrak{s l}}_{2}}(\ell, 0), \omega\right)=\operatorname{Sc}\left(L_{\widehat{\mathfrak{s l}}_{2}}(\ell, 0), \omega\right)=\{\omega, 0\} .
$$

Proof. According to Proposition 3.1, for each semi-conformal vector $\omega_{A}$ of $V_{\widehat{\mathfrak{s}}_{2}}(1,0)$, it can be determined by a symmetric matrix $A$. Since any symmetric matrix can be diagonalized by an orthogonal matrix. Just for every automorphism $\sigma$ of $V_{\widehat{\mathfrak{s l}}_{2}}(1,0)$, its action on $\omega_{A}$ will give a semi-conformal vector $\omega_{o A o^{t r}}$ for $o \in \mathrm{SO}_{3}(\mathbb{C})$. Thus, any semi-conformal vector $\omega_{A}$ can be changed into a semi-conformal vector determined by a diagonal matrix by using some automorphism of $V_{\widehat{\mathfrak{s l}}_{2}}(1,0)$. According to [DL, Proposition 13.10], two conformal vectors are identified in vertex operator algebras $\left(V_{\widehat{\mathfrak{h}}}(1,0), \omega_{\mathfrak{h}}=\frac{1}{2} \alpha_{3}(-1)^{2} \mathbf{1}\right)$ and $\left(V_{\widehat{\mathfrak{s l}}_{2}}(1,0), \omega\right)$, so $\left(V_{\widehat{\mathfrak{h}}}(1,0), \omega_{\mathfrak{h}}\right) \hookrightarrow\left(V_{\widehat{\mathfrak{s l}}_{2}}(1,0), \omega\right)$ is a conformal embedding, that is, $\omega_{A_{3}}=\omega$, where $V_{\widehat{\mathfrak{h}}}(1,0)$ is Heisenberg VOA generated by $\alpha_{3}$. Since $\omega_{A_{1}}=\omega_{M_{1} A_{3} M_{1}^{t r}}$ and $\omega_{A_{2}}=\omega_{M_{2} A_{3} M_{2}^{t r}}$ for some $M_{1}, M_{2} \in \mathrm{SO}_{3}(\mathbb{C})$. Hence $\omega_{A_{4}}=\omega_{A_{5}}=\omega_{A_{6}}=0$. By Proposition 3.11, $V_{\widehat{\mathfrak{s l}}_{2}}(1,0)$ and $L_{\widehat{\mathfrak{s l}}_{2}}(1,0)$ have both only two trivial semi-conformal vectors $\omega$ and 0 .

Remark 4.4. In general, one question is to study the $G$-orbit structure of the variety $\operatorname{Sc}\left(L_{\widehat{\mathfrak{g}}}(1,0), \omega\right)$ for the simple affine vertex operator algebra $L_{\widehat{\mathfrak{g}}}(1,0)$ associated to the finite dimensional simple Lie algebra $\mathfrak{g}$ of $A, D, E$ types. In these cases, we note that $L_{\widehat{\mathfrak{g}}}(1,0) \cong V_{Q_{\mathfrak{g}}}$ as vertex operator algebras, and then we can study $G$-orbit informations of $\operatorname{Sc}\left(L_{\widehat{\mathfrak{g}}}(1,0), \omega\right)$ by virtue of the lattice vertex operator algebra $V_{Q_{\mathfrak{g}}}$ associated to the root lattice $Q_{\mathfrak{g}}$ of $\mathfrak{g}$.

Next, we consider how to determine the order relation of two semi-conformal vectors of $\left(V_{\widehat{\mathfrak{s l}}_{2}}(\ell, 0), \omega\right)$ by their corresponding symmetric matrices. Since any symmetric matrix can be congruent with a diagonal matrix by a special orthogonal matrix, then we only need to consider the order relation of two diagonal matrices.

Proposition 4.5. For two diagonal matrices

$$
A=\left(\begin{array}{lll}
a_{1} & 0 & 0 \\
0 & a_{2} & 0 \\
0 & 0 & a_{3}
\end{array}\right), B=\left(\begin{array}{lll}
b_{1} & 0 & 0 \\
0 & b_{2} & 0 \\
0 & 0 & b_{3}
\end{array}\right)
$$


two vectors $\omega_{A}, \omega_{B} \in \operatorname{Sc}\left(V_{\widehat{\mathfrak{s l}}_{2}}(\ell, 0), \omega\right)$, then $\omega_{A} \preceq \omega_{B}$ if and only if $A, B$ satisfy the following conditions

$$
\left\{\begin{array}{l}
2\left(a_{1} b_{2}+a_{2} b_{1}+a_{1} b_{3}+a_{3} b_{1}-a_{2} b_{3}-a_{3} b_{2}\right)+2 \ell a_{1} b_{1}=a_{1} \\
2\left(a_{1} b_{2}+a_{2} b_{1}+a_{2} b_{3}+a_{3} b_{2}-a_{1} b_{3}-a_{3} b_{1}\right)+2 \ell a_{2} b_{2}=a_{2} \\
2\left(a_{1} b_{3}+a_{3} b_{1}+a_{2} b_{3}+a_{3} b_{2}-a_{1} b_{2}-a_{2} b_{1}\right)+2 \ell a_{3} b_{3}=a_{3} \\
2 \ell a_{1} b_{1}+4 b_{1} a_{2}+4 b_{3} a_{1}-4 b_{3} a_{2}=2 \ell a_{1}^{2}+4 a_{1} a_{2}+4 a_{1} a_{3}-4 a_{2} a_{3} \\
2 \ell a_{2} b_{2}+4 b_{2} a_{1}-4 b_{3} a_{1}+4 b_{3} a_{2}=2 \ell a_{2}^{2}+4 a_{1} a_{2}+4 a_{2} a_{3}-4 a_{1} a_{3} \\
2 \ell a_{3} b_{3}+4 b_{3} a_{1}+4 b_{2} a_{3}-4 b_{2} a_{1}=2 \ell a_{3}^{2}+4 a_{1} a_{3}+4 a_{2} a_{3}-4 a_{1} a_{2} \\
b_{1} a_{2}-b_{2} a_{1}+b_{3} a_{1}-b_{1} a_{3}+b_{2} a_{3}-b_{3} a_{2}=0 .
\end{array}\right.
$$

Proof. According to Proposition [CL, Proposition 2.8], we can verify relations (4.5) by straightly computations .

Next, we expect to describe $\operatorname{MinSc}\left(V_{\widehat{\mathfrak{s l}}_{2}}(\ell, 0), \omega\right)$ for level $\ell \neq-2,1,0,4$.

Theorem 4.6. For the level $\ell \neq-2,0,1,4$, we have

$$
\operatorname{MinSc}\left(V_{\widehat{\mathfrak{s l}}_{2}}(\ell, 0)\right)=\operatorname{Orb}_{A_{1}} \cup \operatorname{Orb}_{A_{4}} .
$$

In particular, when the level $\ell \in \mathbb{Z}_{+}$and $\ell \notin 1,4$, we have

$$
\operatorname{MinSc}\left(L_{\widehat{\mathfrak{s l}}_{2}}(\ell, 0)\right)=\operatorname{Orb}_{A_{1}} \cup \operatorname{Orb}_{A_{4}} .
$$

Proof. We can check $\omega_{A_{1}}$ and $\omega_{A_{4}}$ both don't satisfy the relations (4.5) in Proposition 4.5. So there is not the partial order relation $\preceq$ for the pair $\omega_{A_{1}}, \omega_{A_{4}}$. According to Proposition 3.10, we know that all semi-conformal vectors in a same orbit have the same partial order. Then $\operatorname{Orb}_{A_{1}}, \operatorname{Orb}_{A_{4}} \in \operatorname{MinSc}\left(V_{\widehat{\mathfrak{s l}}_{2}}(\ell, 0)\right)$. Using Corollary 4.2, we have $\operatorname{MinSc}\left(V_{\widehat{\mathfrak{s l}}_{2}}(\ell, 0)\right)=\operatorname{Orb}_{A_{1}} \cup \operatorname{Orb}_{A_{4}}$.

\section{SEmi-CONFORMal VeCtors OF $V_{\widehat{\mathfrak{s l}}_{2}}(4,0)$}

In this section, we consider semi-conformal vectors of $\left(V_{\widehat{\mathfrak{s}}_{2}}(4,0), \omega\right)$. According to equations (4.4), for a diagonal matrix

$$
A=\left(\begin{array}{lll}
a_{1} & 0 & 0 \\
0 & a_{2} & 0 \\
0 & 0 & a_{3}
\end{array}\right),
$$

Note that $\omega_{A} \in \operatorname{Sc}\left(V_{\widehat{\mathfrak{s}}_{2}}(4,0)\right)$ if and only if $A$ satisfies

$$
\begin{aligned}
& 4\left(a_{1} a_{2}+a_{1} a_{3}-a_{2} a_{3}\right)+8 a_{1}^{2}=a_{1} ; \\
& 4\left(a_{1} a_{2}+a_{2} a_{3}-a_{1} a_{3}\right)+8 a_{2}^{2}=a_{2} ; \\
& 4\left(a_{1} a_{3}+a_{2} a_{3}-a_{1} a_{2}\right)+8 a_{3}^{2}=a_{3} .
\end{aligned}
$$


When $A$ satisfies $a_{1}=a_{2}=a_{3}$, the equations (5.1)-(5.3) have two solutions $a_{1}=a_{2}=$ $a_{3}=0$ and $a_{1}=a_{2}=a_{3}=\frac{1}{12}$, i.e., $A=0$ and $A=\frac{1}{12} I$, where $I$ is the $3 \times 3$ identity matrix. They are corresponding to semi-conformal vectors 0 and $\omega$ of $\left(V_{\widehat{\mathfrak{s l}}_{2}}(4,0)\right)$, respectively.

Proposition 5.1. When $a_{1}, a_{2}, a_{3}$ don't satisfy $a_{1}=a_{2}=a_{3}$, the set of solutions of the equations (5.1)-(5.3) is equal to

$$
\left\{\left(a_{1}, a_{2}, a_{3}\right) \in \mathbb{C}^{3} \mid a_{1}+a_{2}+a_{3}=\frac{1}{8} \text { and } a_{1} a_{2}+a_{1} a_{3}+a_{2} a_{3}=0\right\} .
$$

Proof. We solve the equations (5.1)-(5.3) in following cases:

1) If only two numbers of $a_{1}, a_{2}, a_{3}$ are same, we can suppose that $a_{1}=a_{2} \neq a_{3}$, the equations (5.1) and (5.2) both become $12 a_{1}^{2}=a_{1}$, then $a_{1}=0$ or $a_{1}=\frac{1}{12}$.

When $a_{1}=0$, by the equation (5.3), we have $a_{3}=\frac{1}{8}$. So we get a solution $a_{1}=a_{2}=0, a_{3}=\frac{1}{8}$. Since any permutation of $\left(a_{1}, a_{2}, a_{3}\right)$ give a solution of the equations (5.1) and (5.3), then $a_{1}=a_{3}=0, a_{2}=\frac{1}{8}$ and $a_{2}=a_{3}=0, a_{1}=\frac{1}{8}$ are both solutions of the equations (5.1) and (5.3) in this case.

When $a_{1}=\frac{1}{12}$, by the equation (5.3), we have $8 a_{3}^{2}-\frac{1}{3} a_{3}-\frac{1}{36}=0$, so $a_{3}=\frac{1}{12}$ or $-\frac{1}{24}$. In this case, $a_{3}=-\frac{1}{24}$. Hence we get a solution $a_{1}=a_{2}=\frac{1}{12}, a_{3}=-\frac{1}{24}$. Since any permutation of $\left(a_{1}, a_{2}, a_{3}\right)$ give a solution of the equations (5.1) and (5.3) , then $a_{1}=a_{3}=\frac{1}{12}, a_{2}=-\frac{1}{24}$ and $a_{2}=a_{3}=\frac{1}{12}, a_{1}=-\frac{1}{24}$ are another two solutions of the equations (5.1) and (5.3) in this case.

2) Assume that $a_{1}, a_{2}$ and $a_{3}$ are all distinct. Note that $a_{1} \neq a_{2}$, when (5.1) minus (5.2), we have

$$
8\left(a_{1}-a_{2}\right)\left(a_{1}+a_{2}+a_{3}\right)=a_{1}-a_{2},
$$

so we get

$$
a_{1}+a_{2}+a_{3}=\frac{1}{8}
$$

Adding (5.1) and (5.2) to (5.3), we get

$$
8\left(a_{1} a_{2}+a_{1} a_{3}+a_{2} a_{3}\right)+8\left(a_{1}^{2}+a_{2}^{2}+a_{3}^{2}\right)=a_{1}+a_{2}+a_{3},
$$

i.e.,

$$
8\left(a_{1}+a_{2}+a_{3}\right)^{2}-8\left(a_{1} a_{2}+a_{1} a_{3}+a_{2} a_{3}\right)=a_{1}+a_{2}+a_{3} .
$$

Using the relation (5.5), we get

$$
a_{1} a_{2}+a_{1} a_{3}+a_{2} a_{3}=0 .
$$

The solutions of the equations (5.1)-(5.3) given in case 1) all satisfy relations (5.5) and (5.6). 
Conversely, when $a_{1}, a_{2}, a_{3}$ don't satisfy $a_{1}=a_{2}=a_{3}$, using relations (5.5) and (5.6), we can also get the equations (5.1) $-(5.3)$.

Let $S_{3}$ be the permutation group consisting of permutations of $\left(a_{1}, a_{2}, a_{3}\right)$. We have

\section{Theorem 5.2.}

$$
\begin{gathered}
\operatorname{Sc}\left(V_{\widehat{\mathfrak{s}}_{2}}(4,0), \omega\right)=\{0\} \cup\{\omega\} \cup \underset{A \in T}{\bigcup} \operatorname{Orb}_{\omega_{A}}, \\
\text { where } T=\left\{A=\left(\begin{array}{ccc}
a_{1} & 0 & 0 \\
0 & a_{2} & 0 \\
0 & 0 & a_{3}
\end{array}\right) \mid \begin{array}{c}
\text { non - scalar matrix A satisfies } \\
\text { the equations (15.5) and (15.6) }
\end{array}\right\} / S_{3} .
\end{gathered}
$$

Proof. When $A$ is a scalar matrix as a solution of the equations (5.1)-(5.3), we know that $A=0$ or $A=\frac{1}{12} I$, they are corresponding to semi-conformal vectors 0 and $\omega$ of $\left(V_{\widehat{\mathfrak{s l}}_{2}}(4,0), \omega\right)$, respectively. Hence they give two orbits $\{0\}$ and $\{\omega\}$ of $\operatorname{Sc}\left(V_{\widehat{\mathfrak{s l}}_{2}}(4,0), \omega\right)$.

When $A$ is not a scalar matrix as a solution of the equations (5.1)-(5.3), by Proposition 5.1, if and only if $A$ is a solution of the equations (5.5) and (5.6). For any solution $A$ of the equations (5.1)-(5.3), a matrix $B$ given by the action on $A$ of any permutation of $\left(a_{1}, a_{2}, a_{3}\right)$ is still a solution of the equations (5.1)-(5.3). Moreover, $B$ determines the same orbit with $A$ in $\operatorname{Sc}\left(V_{\widehat{\mathfrak{s l}}_{2}}(4,0), \omega\right)$. Hence, we can classify the set of solutions of the equations (5.5) and (5.6) by the action of $S_{3}$. Denote this classified set by $T$. Each element $A$ in $T$ determines a unique orbit $\operatorname{Orb}_{\omega_{A}}$ of $\operatorname{Sc}\left(V_{\widehat{\mathfrak{s l}}_{2}}(4,0), \omega\right)$.

Thus, we get the decomposition of orbits of $\operatorname{Sc}\left(V_{\widehat{\mathfrak{s l}}_{2}}(4,0), \omega\right)$.

As a result of Theorem 5.2, we can get immediately

\section{Corollary 5.3.}

$$
\operatorname{MinSc}\left(V_{\widehat{\mathfrak{s l}}_{2}}(4,0), \omega\right)=\bigcup_{A \in T} \operatorname{Orb}_{\omega_{A}},
$$

where $T$ is the same as in Theorem 5.2.

Proof. According to Proposition 3.8 and Proposition 3.10 , we need only to consider semiconformal vectors determined by diagonal matrices. Given

$$
A=\left(\begin{array}{lll}
a_{1} & 0 & 0 \\
0 & a_{2} & 0 \\
0 & 0 & a_{3}
\end{array}\right), B=\left(\begin{array}{lll}
b_{1} & 0 & 0 \\
0 & b_{2} & 0 \\
0 & 0 & b_{3}
\end{array}\right)
$$

such that $\omega_{A}, \omega_{B} \in \operatorname{Sc}\left(V_{\widehat{\mathfrak{s l}}_{2}}(4,0), \omega\right)$ and they are not equal to $0, \omega$. Then

$$
a_{1}+a_{2}+a_{3}=\frac{1}{8} ; a_{1} a_{2}+a_{1} a_{3}+a_{2} a_{3}=0 \text { and } b_{1}+b_{2}+b_{3}=\frac{1}{8} ; b_{1} b_{2}+b_{1} b_{3}+b_{2} b_{3}=0 \text {. }
$$


According to Proposition 4.5, we can check that $A, B$ don't satisfy the relations (4.5), hence $\omega_{A}$ and $\omega_{B}$ don't have partial order relation, so $\operatorname{MinSc}\left(V_{\widehat{\mathfrak{s l}}_{2}}(4,0), \omega\right)$ consists of nontrivial semi-conformal vectors of $V_{\widehat{\mathfrak{s l}}_{2}}(4,0)$.

Corollary 5.4. The set $T$ in Corollary 5.3 is an algebraic variety isomorphic to the affine line $\mathbb{A}^{1}$. There are infinitely many $\mathrm{SO}_{3}(\mathbb{C})$-orbits in $\operatorname{MinSc}\left(L_{\widehat{\mathfrak{s}}_{2}}(4,0), \omega\right)$.

Proof. Note that two diagonal matrices $A$ and $B$ are in the same $\mathrm{SO}(\mathbb{C})$-orbit if any only they are equal up to permutation of the diagonal entries. Consider the affine variety, $\mathbb{C}^{3}$, the set $\mathbb{C}^{3} / S_{3}$ is identified by the affine variety with the coordinate algebra being the algebra of symmetric polynomials $\mathbb{C}\left[x_{1}, x_{2}, x\right]^{S_{3}}=\mathbb{C}\left[e_{1}, e_{2}, e_{3}\right]$, which is a polynomial algebra. Here $e_{1}, e_{2}$, and $e_{3}$ are the elementary symmetric polynomials. Then the set $\operatorname{MinSc}\left(L_{\widehat{\mathfrak{s}}_{2}}(4,0), \omega\right) / G=T$ which is defined by the equations $e_{\frac{1}{8}}$ and $e_{2}=0$. In particular, $T$ is an algebraic variety isomorphic to the affine line $\mathbb{A}^{1}$.

Remark 5.5. Comparing to the cases of $\ell \neq-2,0,1$, we know that $\operatorname{MinSc}\left(V_{\widehat{\mathfrak{s l}}_{2}}(4,0), \omega\right)$ can be expressed as the union of infinite $\mathrm{SO}_{3}(\mathbb{C})$-orbits, i.e.,

$$
\operatorname{MinSc}\left(V_{\widehat{\mathfrak{s l}}_{2}}(4,0), \omega\right)=\bigcup_{\omega_{A} \in T} \operatorname{Orb}_{\omega_{A}},
$$

where $\operatorname{Orb}_{\omega_{A}}=\left\{\omega_{o A o^{t r}} \mid o \in \mathrm{SO}_{3}(\mathbb{C})\right\}$ and $T$ is the same as in Theorem 5.2 .

Remark 5.6. By Proposition 3.11, we know that

$$
\operatorname{Sc}\left(L_{\widehat{\mathfrak{s}}_{2}}(4,0), \omega\right)=\operatorname{Sc}\left(V_{\widehat{\mathfrak{s}}_{2}}(4,0), \omega\right)
$$

and

$$
\operatorname{MinSc}\left(L_{\widehat{\mathfrak{s l}}_{2}}(4,0), \omega\right)=\operatorname{MinSc}\left(V_{\widehat{\mathfrak{s}}_{2}}(4,0), \omega\right)
$$

The following result is obtained from above theorem immediately.

Corollary 5.7. For the level $\ell \neq-2,0,1$, there exists a maximal chain of partial order in $\operatorname{Sc}\left(V_{\widehat{\mathfrak{s}}_{2}}(\ell, 0), \omega\right)$ (resp. $\operatorname{Sc}\left(L_{\widehat{\mathfrak{s l}}_{2}}(\ell, 0), \omega\right)$ for $\ell \in \mathbb{Z}_{+}$and $\left.\ell \neq 1\right)$

$$
0=\omega^{0} \prec \omega^{1} \prec \omega^{2}=\omega
$$

with the length 2.

Remark 5.8. Our aim is to understand vertex operator algebra theory by the geometric object $\operatorname{Sc}(V, \omega)$. For this purpose, at first, we must understand clearly the structures of $\operatorname{Sc}(V, \omega)$. From above results related to the affine vertex operator algebra $\left(V_{\widehat{\mathfrak{s l}}_{2}}(\ell, 0)\right.$, $\left.\omega\right)$, we can find the variety $\operatorname{Sc}(V, \omega)$ is closely related to the level $\ell$ for the affine vertex operator algebra $\left(V_{\widehat{\mathfrak{s l}}_{2}}(\ell, 0), \omega\right)$, and this work will lead us to describe $\operatorname{Sc}(V, \omega)$ for general affine vertex operator algebras and some even lattice vertex operator algebras. In future work, 
we shall focus on cases for general affine vertex operator algebras and some even lattice vertex operator algebras and discuss structures and properties of the varieties $\operatorname{Sc}(V, \omega)$ to understand vertex operator algebra theory.

\section{REFERENCES}

[BF] D. Ben-Zvi, E. Frenkel, Vertex algebras and algebraic curves, 2nd ed., Mathematical Surveys and Monographs 88, Amer. Math. Soc. 2004.

[CHZ] Y. Chu, F. Huang, Z. Zheng, A commutant of $\beta \gamma$-system associated to the highest weight module $V_{4}$ of $\mathfrak{s l}(2, \mathbb{C})$, J. Math. phys,51(2010), 092301-01-32.

[CL] Y. Chu, Z. Lin, The varieties of Heisenberg vertex operator algebras. Science China Math. Vol.60, no. 3(2017), 379-400.

[D] C. Dong, Representations of the moonshine module vertex operator algebra, Contemp. Math. 175(1994), 27-36.

[DL] C. Dong, J. Lepowsky, Generalized Vertex Algebras and Relative Vertex Operators. Progress in Math, Vol 112. Boston: Birkhauser, 1993.

[DLinM] C. Dong, Z. Lin, G. Mason, On vertex operator algebras as sl $_{2}$-modules, in: Groups, difference sets, and the Monster (Columbus, OH, 1993), Ohio State University Mathematics Reserach Institute Publication, Vol. 4, de Gruyter, Berlin, 1996, 349-362.

[DLM] C. Dong, H. Li, G. Mason, Some twisted modules for the moonshine vertex operator algebras, Contemp. Math. 193 (1996), 25-43.

[DLMM] C. Dong, H. Li, G. Mason and P. Montague, The radical of a vertex operator algebra, in: Proc. of the Conference on the Monster and Lie algebras at The Ohio State University, May 1996, ed.

[DLMN] C. Dong, H. Li, G. Mason and S. P. Notton, Associative subalgebras of the Griess algebra and related topics, in "The Monster and Lie algebras" (J. Ferrar and K. Harada, Eds.) de Gruyter, Berlin, 1998, pp. 27-42.

[DLY] C. Dong, C. H. Lam, H. Yamada, W-algebras related to parafermion algebras, J. Alg. 322 (2009), 2366-2403.

[DM] C. Dong and G. Mason, On quantum Galois theory, Duke Math. J. 86(1997),305-321.

[DMZ] C. Dong, G. Mason and Y. Zhu, Discrete series of the Virasoro algebra and the moonshine module, In: Algebraic groups and their generalizations: quantum and infinite-dimensional methods, Proc. Symp. Pure. Math. American Math. Soc. 56 II (1994), 295-316.

[DW1] C. Dong, Q. Wang, The Structure of Parafermion vertex operator algebras: general case, Comm. Math. Phys. Vol. 299 (2010), 783-792.

[DW2] C. Dong, Q. Wang, Parafermion vertex operator algebras, Front. Math. China, 6, no.4(2011), 567-579.

[DZ] C. Dong, Wei Zhang, Rational vertex operator algebras are finitely generated, J. Alg. 320 (2008), 2610-2614.

[FHL] I. B. Frenkel, Y. Huang and J. Lepowsky, On axiomatic approaches to vertex operator algebras and modules, Memoirs American Math. Soc. 104, 1993.

[FLM] I. B. Frenkel, J. Lepowsky, A. Meurman, Vertex operator algebras and the Monster, Pure and Applied Math. Vol. 134, Academic Press, Boston, 1988. 
[FZ] I. B. Frenkel and Y. Zhu, Vertex operator algebra associated to representations of affine and Virasoro algebras, Duke Math. J. 66(1992), 123-168.

[H] Y. Huang, A non-meromorphic extension of the moonshine module vertex operator algebra, Contemp. Math. 163 (1996), 123-148.

[Ho] G. Hohn, The group of symmetries of the shorter moonshine module, Abh. Math. Semin. Univ. Hambg. 80, no.2 (2010), 275-283.

[JL1] C. Jiang, Z. Lin, The commutant of $L_{\widehat{\mathfrak{s l}_{2}}}(n, 0)$ in the vertex operator algebra $L_{\widehat{\mathfrak{s l}_{2}}}(1,0)^{\otimes n}, A d v$. in Math. 301 (2016), 227-257.

[JL2] C. Jiang, Z. Lin, Tensor decomposition, parafermions, level-rank duality, and reciprocity law for vertex operator algebras, arXiv:1406.4191

[K] V. Kac, Vertex algebras for beginners, 2nd ed., University Lecture Series, Vol.10, Amer. Math. Soc., Providence, RI, 1998.

[KL] M. Kitazume, C. H. Lam, Decomposition of the moonshine vertex operator algebra as Virasoro modules, J. Alg. 226(2000), 893-919.

[L] C. H. Lam, Code vertex operator algebras under coordinates change, Comm. Alg. 27, no.9 (1999), 4587-4605.

[Li] H. Li, Some finiteness properties of regular vertex operator algebras, J. Alg. 212 (1999), 495-514.

[LL] J. Lepowsky and H. Li, Introduction to vertex operator algebras and their representations, Progress in Mathematics, Vol. 227, Birkhser Boston, Inc., Boston, MA, 2004.

[LL1] B. Lian, A. Linshaw, Howe Pairs in the theory of Vertex algebras. J. Alg. 317, 111(2007).

[LK] H. Li, M. Karel, Certain generating subspace for vertex operator algebras, J. Alg. 217 (1999), 393-421.

[LS] C. H. Lam and H. Shimakura, Ising vectors in the vertex operator algebra $V_{\Lambda}^{+}$associated with the Leech lattice $\Lambda$, Int. Math. Res. Not. 2007, no. 24 (2007), Art. ID rnm 132, 21p.

[LSY] C. H. Lam, S. Sakuma, and H. Yamauchi, Ising vectors and automorphism groups of commutant subalgebras related to root systems, Math. Zeit. 255, no.3 (2007), 597-626.

[LY] C. H. Lam, H. Yamada, Decomposition of the lattice vertex operator algebra $V_{\sqrt{2} A_{l}}, J$. Alg. 272 (2004), 614-624.

[M] M. Miyamoto, Griess algebras and conformal vectors in vertex operator algebras, J. Alg. 179, no.2(1996), 523-548.

[S] S. Sakuma, 6-transposition property of $\tau$ - involutions of vertex operator algebras, Int. Math. Res. Not. 2007, no. 9 (2007), Art. ID rnm 030, 19 p.

[Sh] H. Shimakura, Classification of Ising vectors in the vertex operator algebra $V_{L}^{+}$, Pacific J. Math. 258, no. 2(2012), 487-495.

(Chu) 1.School of Mathematics and Statistics, Henan University, Kaifeng, 475004, China, 2. Institute of Contemporary Mathematics, Henan University, Kaifeng 475004, CHINA

E-mail address: chuyj@henu.edu.cn

(Lin) Department of Mathematics, Kansas State University, Manhattan, KS 66506, USA

E-mail address: zlin@math.ksu.edu 